University of Zurich

Department of Economics

Working Paper Series

ISSN 1664-7041 (print)

ISSN 1664-705X (online)

Working Paper No. 320

\title{
Market power and information effects in a multi-unit auction
}

Andreas Hefti, Peiyao Shen and Regina Betz

March 2019 


\title{
Market power and information effects in a multi-unit auction
}

\author{
Andreas Hefti, Peiyao Shen and Regina Betz*
}

\begin{abstract}
We study the effects of different information structures (full information, supply uncertainty and demand uncertainty) on equilibrium prices, allocative efficiency and bidding behavior in a (supply-side) uniform-price multi-unit auction, using supply function competition and a novel experimental design. Our setup integrates different types of market power and a varying level of competition. We empirically find that average prices tend to be higher under full information compared to the cases where bidders either have limited information about about the demand level or rivals' technologies or; the latter even leading to strictly lower average prices as the exertion of market power and bid shading is strongly reduced. We explain this finding with a behavioral equilibrium concept, where bidders behave as if competing against the average market situation. Further, we address the problem of multiplicity of equilibria by exploiting the equilibrium conditions to obtain an empirical selection of the average equilibrium supply function. The respective predictions of the average prices exceed those by standard OLS in all information treatments.
\end{abstract}

Keywords: Multi-unit auctions; Limited Information; Market Power; Supply function competition; Supply uncertainty; Demand uncertainty; Restricted Least Squares

JEL classification: C92;D43;D44;D82;L11;L94;Q41

First version: Nov 2018

This version: March 2019

\footnotetext{
${ }^{*}$ Corresponding author: Andreas Hefti, Blumlisalpstr. 10, CH-8006 Zurich. Email: andreas.hefti@econ.uzh.ch. Author affiliations: Hefti: I) Department of Economics, University of Zurich, II) School of Management and Law, Zurich University of Applied Sciences. Shen: School of Entrepreneurship and Management, ShanghaiTech University. Betz: School of Management and Law, Zurich University of Applied Sciences.

Our special thanks go to Marek Pycia. We thank Iain MacGill, David Salant, Jakub Steiner, Ricardo Gonçalves, Xavier Vives, Samuel Haefner, and the audience at presentations at UECE Games 2018, EAERE 2017, Swissgrid, ELCOM, UZH and ZHAW for helpful comments and enlightening discussions. The authors acknowledge financial support from the SCCER CREST (Swiss Competence Center for Research in Energy, Society and Transition) managed by the Swiss Innovation Agency (Innosuisse) under Grant No. 1155002547. Andreas thanks Ines Brunner for support.
} 


\section{Introduction}

In many real-world markets auctions are the main mechanisms implemented to allocate multiple units of an asset across bidders. Examples range from the private to the public sector, including wholesale electricity markets, electromagnetic spectrum, treasury and central bank liquidity auctions, refinancing, financial markets, real estate and pollution permits. The stakes at such auctions are frequently tremendous. ${ }^{1}$ Market power of certain bidders is of great practical relevance in many of these cases. For example, the top-five bidders in U.S. Treasury auctions were estimated to acquire up to half of the issue, despite a large overall number of participants (Malvey and Archibald, 1998). Armantier and Sbai (2006), Hortaçsu and McAdams (2010), Kastl (2011), Cassola et al. (2013) and Hortaçsu et al. (2018) provide similar evidence. In electricity markets, Borenstein et al. (2002) attributed up to half of the increase in California's electricity expenditure (from $2 b \$$ to $9 b \$$ ) observed in the period 1999 - 2000 to bidders with market power. Similar findings are reported by Wolfram (1998) for the British electricity market, and by Hortaçsu and Puller (2008) for the Texas electricity market. ${ }^{2}$ Bidder-side market power was also reported in procurement markets, for instance for school milk (Pesendorfer, 2000).

Tirole (1988) loosely describes market power as an agent's ability to "make the price", where such market power may arise for various reasons. Some agents may have market power because they face favorable conditions for their actions compared to others. Examples comprise of a cheaper production technology, larger production capacities, lower transaction costs, scale advantages or heterogeneous preferences. Such advantages may allow to influence the price in one's favor. A wellknown and extreme instance is Bertrand competition with asymmetric firms, where the lowest-cost firm fully exploits her "exclusive" market power by seizing the entire market at a price equal to the marginal cost of the next-best competitor (Blume, 2003). A different form of market power arises if some agents are pivotal for market clearing. Pivotal bidders have an incentive to shade their bids in order to increase the equilibrium price. Such type of market power matters in electricity markets, and multi-unit auctions more generally (Ockenfels, 2007; von der Fehr, 2013; Brandts et al., 2014).

A central aspect related to market power is the bidder-side information about the market. The relevant information set for a bidder can be roughly decomposed into information about idiosyncratic and aggregate market conditions. The cost or valuation structure of all bidders in the

\footnotetext{
${ }^{1}$ For example, the value of the US Treasury auctions in 2013 was 7.9 Trillion USD (Hortaçsu et al., 2018), or the monetary value of 356 auctions (from 1980 to 2014) of government bonds on behalf of the Swiss treasury is 149 '435 Billion CHF (Ranaldo and Rossi, 2016). Likewise, the value in Australia's National Electricity Market (NEM) from 2016-2017 was 16.6 Billion AUD (https://www.aemo.com.au/Electricity/National-Electricity-Market-NEM).

${ }^{2}$ Also see Joskow and Kahn (2002); Wolak (2003); Ockenfels (2007); Bosco et al. (2012); von der Fehr (2013); Dormady (2014); Holmberg and Wolak (2015) on the empirical relevance of market power in electricity markets.
} 
market, or the number of competitors active in certain price ranges are examples for the former, while the overall level of demand in a supply-side auction is an example for the latter. Real-world multi-unit auctions may differ in the information bidders have about such aspects. Moreover, the prevailing information structure may change as a consequence of various current and near-future developments. First, the developments in IT and computation may affect the quality of the individually available information. For example, it is conceivable that artificial intelligence or big-data mining can improve bidder-side information, and therefore reduce some uncertainty in markets about idiosyncratic or aggregate conditions. Second, structural change may also affect the prevailing information structure. Future electricity markets, for instance, most likely will feature a much larger share of renewable energy generation, the supply of which depends directly on certain weather patterns. ${ }^{3}$ Compared to current and past energy markets, which are typically thought of as rather close to complete information, ${ }^{4}$ such a development adds a new source of uncertainty about the effective number of competitors, their capacities and their supply costs. Third, the information available to bidders may be affected by regulation. For example, the European Union has recently adopted the Regulation on Wholesale Energy Market Integrity and Transparency (REMIT, No 1227/2011), which requires individual facilities to make their planned and unplanned capacity outages, e.g., due to maintenance, available to other bidders.

It is a largely open question how limited bidder information, or changes therein, affect market power and strategic bidding in multi-unit auctions. In this paper we therefore study how strategic bidding and equilibrium outcome in a supply-side multi-unit auction depend on market power, varying levels of competition, and different information structure. In a nutshell, we seek to learn if individual bidding becomes more or less competitive once bidders know more or less about the other bidders or the number of units at auction. To this end, we construct an experimental design that has the information structure as its main treatment variable, and allows for various bidder constellations generating different levels of competition and market power. We concentrate on the case of a sealed-bid seller-side uniform-price auction, and use the theory of supply function competition to obtain a set of equilibrium predictions, which we can test in the data. The concept of supply function competition fits closely with our experimental design, where participants enter their quantities for the various possible price levels, thereby constructing a supply function. We concentrate on the uniform-price auction because this is the prevalent auction format in many

\footnotetext{
${ }^{3}$ See, e.g., International Energy Agency 2016: World Energy Investment 2016, Executive Summary: https: //www . iea.org/Textbase/npsum/WEI2016SUM. pdf.

${ }^{4}$ See, e.g., Hortaçsu and Puller (2008). These authors also note that already current energy markets may feature more idiosyncratic uncertainty than commonly assumed, e.g., because of different forward contracts that are only privately known.
} 
cases. $^{5}$

In our framework, bidders can differ in their available production technology (costs and capacities). Further, the demand level, i.e., the total number of units that bidders can sell, may vary. The parameters of the design allow for situations with exclusive and pivotal market power, as well as for a varying degree of competition as measured by the number of identical bidders. Depending on the information structure, bidders may have full or only statistical information about the situation at hand. In particular, our design distinguishes between the cases of complete information, asymmetric information about the technologies (costs and capacities) of the other bidders, and identical but incomplete information about the level of demand.

This article makes a threefold contribution. First, we find that if demand is common knowledge but own capacity and supply costs are bidders' private information - a situation we refer to as supply uncertainty - the empirical auction prices, averaged over all relevant market situations, are significantly lower compared to the full information case: Supply uncertainty reduces average markups by $13 \%$. The main empirical source of this price decrease is a significant reduction of bid shading in situations that would feature strong market power under full information. Specifically, with supply uncertainty we observe an average reduction of markups by $29 \%$ in situations with purely exclusive market power, and by $56 \%$ in situations with purely pivotal market power. We explain this finding by means of a behavioral equilibrium concept, which we call Average Linear Supply Function Equilibrium (A-LinSFE). In an A-LinSFE, each bidder resolves the uncertainty by acting as if the expected number of competitors were present in the market for sure, while the resulting aspired auction price must be correct on average. We show that the range of average prices (averaged across all relevant market situations) that are supported as equilibrium outcomes is strictly smaller than its full information counterpart. Particularly, the highest full information equilibrium prices are not feasible as outcomes in any A-LinSFE. In this sense, the average-linear thinking embodied in the equilibrium play of A-LinSFE tends to make bidders pessimistic about high prices. The concept of A-LinSFE can also be applied if the auction features demand uncertainty, and offers a similar pattern regarding equilibrium prices. The data confirms that average prices are not higher with demand uncertainty. In contrast to supply uncertainty, however, the data does not allow us to conclude that average prices are strictly lower with demand uncertainty in general, except for situations with symmetric pivotal market power.

Second, the data shows that an increase in competition, as measured by a larger number of symmetric competitors in a market, or a decease in demand have the intuitive effect of decreasing

\footnotetext{
${ }^{5}$ See, e.g., "Power market auction design: rules and lessons in market-based control for the new electricity industry", www.eei.org.
} 
auction prices, independent of the information structure. Adding a second competitor decreases markups by about $50 \%$ on average, and increasing demand by $10 \%$ increases markups by about $16 \%$. Also, we find that average prices are significantly above marginal costs in all information treatments, consistent with a basic prediction of our symmetric linear supply function equilibria. Moreover, in both uncertainty treatments the data mostly corroborates the A-LinSFE prediction that average prices should reside within certain price intervals pooled across those situations which cannot be discerned by the bidders due to their limited information.

Third, we exploit the theoretical restrictions imposed by supply function competition within an otherwise standard OLS framework to obtain an empirical selection of the average equilibrium supply function. Supply function competition can lead to multiple equilibria in many settings, including ours. In situations with two or more symmetric bidders and full information every affine-linear symmetric supply function equilibrium satisfies a uniquely determined constraint that pins down the slope and intercept parameters of the equilibrium supply function with one degree of freedom. A similar result also applies to A-LinSFE in the two uncertainty treatments. The predictions of the average auction prices obtained from a restricted least squares estimation with these constraints outperform their standard OLS counterparts, and in all cases are statistically indistinguishable from the true average prices. Moreover, in both uncertainty treatments we even find that the predicted prices implied by the restricted approach are statistically identical to the true average prices in every market situation that is indistinguishable to bidders given their limited information. We view these observations as additional support for the concept of supply function equilibrium to be a useful framework for studying multi-unit auctions.

\subsection{Related Literature}

Our article contributes to the general literature on information effects in strategic models. ${ }^{6}$ To our knowledge, we are the first to empirically assess the comparative effects of complete information, private cost information and demand uncertainty on bidding behavior and equilibrium outcome in the multi-unit auction context. The vast part of experimental studies on multi-unit auctions are concerned with comparing different auction formats according to various criteria (see, e.g., the survey by Kwasnica and Sherstyuk (2013)). Further, a large portion of the empirical literature on (uniform-price) multi-unit auctions concentrates on eliciting to what extent individual bidders best-respond to each other, while a different strand is concerned with recovering underlying fundamentals, such as costs, from bidding data (see the survey by Hortaçsu and McAdams, 2018). In the single-unit auction case, Andreoni et al. (2007) conduct experimental first- and second price buyer

\footnotetext{
${ }^{6}$ The textbook by Vives (1999) provides a nice introduction.
} 
auctions, where they compare the full information with the private value case. The key theoretical prediction is that there should be no difference in average revenues (and prices) across these two information structures. ${ }^{7}$ Contrary to theory, they empirically find that first-price auction prices (buyer revenues) are higher, and bidding thus more competitive, in the private value case. Related, Buchanan et al. (2016) find that not revealing information about group size and already sold units increases the average price in a Dutch clock auction, where $n$ buyers can acquire at most a single unit of $m$ goods. Both findings resemble our own result that supply uncertainty leads to a more competitive outcome.

On the pure theory side, Vives (2011) in a linear-Gaussian model and Holmberg and Wolak (2015) in a single-bid duopoly are the only papers we are aware of that at least partly compare private and full information in a multi-unit auction context. ${ }^{8}$ Vives (2011) considers (Bayesian) linear supply function competition with private information (supply uncertainty) and possibly also demand uncertainty, where bidder costs can be correlated, and bidders learn their own costs possibly with some noise. His setting allows for a unique equilibrium. ${ }^{9}$ In contrast, we study a setting where multiple equilibria occur, but use the underlying equilibrium restrictions on all equilibria identified by the model to estimate supply functions and equilibrium prices in a constrained regression framework. ${ }^{10}$ One prediction is that if costs are independent, or own costs are observed without noise then, if an equilibrium exists, the Bayesian equilibrium coincides with the full information case. More generally, if there is an effect from changes in the available information, then its main direction is that less information about costs ("more noise") leads to higher prices. ${ }^{11}$ Our experimental setting features independence of cost and demand shocks, but we find that average prices are reduced in the case where costs are (perfect) private information. Finally, Vives (2011) predicts that a decrease in (inelastic) demand or an increase in the number of competitors should lead to lower expected prices, which we confirm theoretically and empirically. ${ }^{12}$

\footnotetext{
${ }^{7}$ Kim and Che (2004) prove revenue equivalence between the private and complete information case for the first-price auction. Morath and Münster (2008) prove the same result for the second-price auction, and for arbitrary convex combinations between the two. Additionally, equivalence of bidder surplus between the two information formats is shown by Bergemann et al. (2017).

${ }^{8}$ Vives (2011) restricts attention to the uniform-price auction; Holmberg and Wolak (2015) also compare the uniform-price to a discriminatory auction format.

${ }^{9}$ Uniqueness of supply function equilibria can be obtained under different conditions (see Klemperer and Meyer, 1989; Holmberg, 2008).

${ }^{10}$ The existence of multiple SFE are a well-known phenomenon, particularly if there is no uncertainty (Klemperer and Meyer, 1989), which is among the information structures we consider.

${ }^{11}$ This may happen with correlated costs and noisy percepts of own costs. The intuition is that, in a Gaussian world, the Bayesian equilibrium is privately revealing as the conditional expectation is a sufficient statistic for the conditional distribution, such that the price begins to act as a "conveyor of information". A high price conveys that costs of other sellers are high and, by correlation, own costs must therefore also be high. Hence, a rational seller reduces her quantity supplied more as the price increases. Less information, in the sense of more noisy signals about costs, therefore tends towards causing higher prices. This channel is entirely absent with independent values. See Holmberg and Wolak (2015) for a similar result.

${ }^{12}$ In our setting, the respective theoretical predictions are more subtle due to multiplicity of equilibria.
} 
Market power has been addressed by a number of papers in multi-unit auction research. ${ }^{13}$ In multi-unit auctions, market power is manifested through differential bid shading of different bidders. Because incentives to shade bids increase in the quantity a bidder seeks to buy (or to sell), bid shading by advantaged bidders, or bidders with pivotal market power, may have a substantial impact on market outcome (Ausubel et al., 2014). Rassenti et al. (2003) find that advantaged sellers exercise their market power in an experimental multi-unit auction, where both demand and bidding costs are common knowledge. Hefti and Shen (2019) examine the case where a bidder has exclusive market power, analogous to Bertrand competition with asymmetric costs, in the same multi-unit auction studied by the present article. Pivotal market power has been studied theoretically in the multi-unit auction context by Genc and Reynolds (2011) and also experimentally by Brandts et al. (2014) in case of demand uncertainty. Genc and Reynolds (2011) consider the case of symmetric pivotal market power, and show that the set of symmetric equilibria is increasing in supply capacities in presence of pivotal suppliers. Brandts et al. (2014) predict that pivotal market power in case of symmetrically pivotal firms and complete cost information relaxes competition by restricting the range of possible supply function equilibria. Their experimental data shows that pivotal market power is exerted; average prices are higher in treatments with pivotal power, a result which our experiment also confirms. ${ }^{14}$ We differ from these studies by considering how changes in the information available to bidders affect the degree of exerted symmetric or asymmetric pivotal market power, and by also considering non-pivotal market power as well as a varying level of competition.

Finally, we contribute to a number of papers arguing that supply function competition describes the empirical bidding behavior well, even if true bidding is "discrete". In particular, Bolle et al. (2013) find that the shape of the supply functions are generally in line with theoretical predictions, but behavior seems sensitive to theoretically irrelevant changes in demand uncertainty. Brandts et al. (2014) find in their experiment with demand uncertainty that the supply function approach tends to outperform the multi-unit auction model (Fabra et al., 2006), e.g., because empirical supply functions are upward sloping and prices above marginal costs absent market power, a finding we also confirm across information treatments. The constraint regression framework invoked by our paper provides additional evidence supporting that supply function competition is a reasonable framework to describe the average bidding behavior.

\footnotetext{
${ }^{13}$ See Davis and Holt (2008) for a general overview of market power research in laboratory experiments.

${ }^{14}$ Dormady (2014) also finds experimental evidence that pivotal market power inflates the average price in a complete information multi-unit auction setting with heterogeneous bidders. Dormady (2016) further emphasizes a problematic link between market power in energy markets and emission permission markets, particularly in times of peak energy demand.
} 


\subsection{Article structure}

Section 2 develops the supply function equilibrium and derives the relevant empirical hypothesis. The experimental design and the empirical results are presented in section 3.

\section{Supply function competition}

We consider a supply-side uniform-price multi-unit auction, where $n>1$ bidders compete over a quantity of $\bar{Q}>0$ units of a good. Bidder $j$ 's technology is the pair $\tau_{j} \equiv\left(c_{j}, k_{j}\right)$, where $c_{j}:\left[0, k_{j}\right] \rightarrow \mathbb{R}_{+}$is a non-decreasing cost function, and $k_{j}>0$ the maximal capacity $j$ can supply to the market. Hence $c_{j}\left(q_{j}\right) \geq 0$ are the costs bidder $j$ incurs from producing and supplying $q_{j} \in\left[0, k_{j}\right]$ units to the market. Every bidder chooses her supply function $q_{j}: \mathcal{P} \rightarrow\left[0, k_{j}\right]$, a nondecreasing, continuous map defined on a compact price space $\mathcal{P} \subset \mathbb{R}_{+}$with smallest and largest element $P=0$ and $P=P_{\max }>0$ (the price cap), respectively. In most of the theoretical part it is analytically convenient to analyze the model by assuming a convex price space $\mathcal{P}=\left[0, P_{\max }\right]{ }^{15}$ A price cap, capacity constraints and an inelastic demand are common characteristics, e.g., of electricity markets (Stoft, 2002). Aggregate supply is $q(P) \equiv \sum_{j=1}^{n} q_{j}(P)$, where $q(\cdot) \in\left[0, \sum_{j} k_{j}\right]$ is non-decreasing and continuous. The auction price $P^{*}$ is the lowest price for which $q(P) \geq \bar{Q}$, or $P^{*}=P_{\max }$ if $q\left(P_{\max }\right)<\bar{Q}$; the corresponding auction allocation is $\left(q_{j}\left(P^{*}\right)\right)_{j}$.

Information structure We differentiate between three prototypical types of information structures: Full information, supply uncertainty and demand uncertainty. Full information means that all technologies $\left(\tau_{1}, \ldots, \tau_{n}\right)$ as well as demand $\bar{Q}$ are common knowledge; hence all bidders play a game of complete information. Supply uncertainty means that technologies are private information, while demand is common knowledge. Hence bidders play a game of asymmetric information, facing uncertainty about the other bidders' technologies. Finally, demand uncertainty means that technologies are common knowledge, but demand is unknown. Hence bidders play a game of symmetric but incomplete information, facing an aggregate uncertainty about demand.

In the experiment, the information structure is the only treatment variable. Thus, the underlying distribution of technologies and market demand remains the same throughout all three information settings. This procedure allows us to elicit the effects of the specific type of uncertainty induced by the corresponding information structures on bidding behavior and auction outcome.

\footnotetext{
${ }^{15}$ Our experiment features a discrete price space with many price steps (from 0 to 100 in steps of $\Delta=5$ ) which we view as approximating the convex-valued case. It is known that a given continuous supply function equilibrium is robust to a "discretization" of the strategy space under certain conditions (Holmberg et al., 2013).
} 


\subsection{Market power and competition}

A market is described by the bidders $j=1, . ., n$ with technologies $\left(\tau_{1}, \ldots, \tau_{n}\right)$, and by the auctioned quantity $\bar{Q}$. Depending on the distribution and specifications of the technologies relative to $\bar{Q}$, two different types of market power can arise in the multi-unit auction context.

Definition 1 (Market power) A bidder has pivotal market power if the market can clear only with his capacity. A bidder has exclusive market power if she can profitably serve the entire market at a price which is not profitable for any other bidder. A market features structural market power if there is a bidder with exclusive or pivotal market power. A market is competitive if it does not feature structural market power.

Our experimental design features both types of market power. Exclusive market power is not a pure multi-unit auction phenomenon. It can arise in single-unit auctions and related models, such as Bertrand competition. ${ }^{16}$ By contrast, there is no single-unit analogue of pivotal market power. Further, one can distinguish between asymmetric or symmetric pivotal market power. A market features symmetric pivotal power if all $n$ bidders are identical $\left(\tau_{1}=\ldots=\tau_{n}\right)$ and their joint capacity clears the market, while the joint capacity of any $n-1$ bidders does not. With symmetric pivotal market power any single bidder is pivotal. Asymmetric pivotal power occurs if not all bidders are identical but the capacity of a certain bidder is necessary for the market to clear. Note that a market may simultaneously feature asymmetric pivotal and exclusive market power, but not symmetric pivotal and exclusive market power.

\section{$2.2 \quad$ Experimental setup}

Conducting a multi-unit auction experiment naturally requires us to parametrize bidder technology. In the following, we outline the type of technology we administer in the experiment. A bidder has one of two possible technologies: $\tau_{L}=\left(c_{L}, \bar{k}\right), \tau_{H}=\left(c_{H}, \underline{k}\right)$, where

$$
c_{L}(q)=\left\{\begin{array}{ll}
\underline{\mathrm{c}}, & q \leq \bar{q} \\
\bar{c}, & \bar{q}<q \leq \bar{k}
\end{array} \quad c_{H}(q)=\bar{c}, \quad q \leq \underline{k},\right.
$$

with $0<\bar{q}<\bar{k}$ and $\underline{k}<\bar{k}$. We refer to a bidder with technology $\tau_{L}, \tau_{H}$ as low-cost and high-cost bidders, respectively. A low-cost bidder is characterized by a larger overall capacity, and can supply at lower marginal costs up to a certain quantity. ${ }^{17}$

\footnotetext{
${ }^{16}$ Exclusive market power in the multi-unit auction context is the main topic of Hefti and Shen, 2019.

${ }^{17}$ Besides its simplicity, the motivation for such a cost-capacity structure comes from wholesale electricity markets. We think of each supplier as endowed with a traditional generator (e.g., gas or coal), which can produce up to $\bar{k}$ units at comparably high marginal costs. Additionally, some bidders may have a renewable generator (e.g., PV or
} 
Whether a market features structural market power depends on bidder technologies and on $\bar{Q}$. In the experimental design, we chose the demand and cost levels such as to obtain the combination of the various possibilities. Specifically, we let $n=3, \bar{Q} \in\{60,100,180\}, \underline{\mathrm{c}}=10, \bar{c}=50, \bar{q}=80$, $\bar{k}=120$ and $\underline{k}=40 .{ }^{18}$ With $n=3$, two technologies and three demand levels, 12 possible market situations emerge. Given the binary nature of technology, the number of low-cost bidders can be used as the distinguishing aspect for the various situations for a fixed $\bar{Q}$. The four possibilities are indicated as $s_{1}$ (only low-cost bidders), $s_{2}$ (two low-cost bidders), $s_{3}$ (one low-cost bidder) and $s_{4}$ (only high-cost bidders), respectively. A specific market situation is labeled by a pair $(s, \bar{Q})$. Table 1 presents an overview of all market situations covered by the experiment, jointly with their type of market power (if any).

Table 1: Experimental market situations: Market power and competition

\begin{tabular}{|c|c|c|c|c|}
\hline & $s_{1}$ & $s_{2}$ & $s_{3}$ & $s_{4}$ \\
\hline $\bar{Q}=60$ & $\mathrm{C}$ & $\mathrm{C}$ & $\begin{array}{c}\text { SMP } \\
\text { exclusive }\end{array}$ & $\mathrm{C}$ \\
\hline $\bar{Q}=100$ & $\mathrm{C}$ & $\mathrm{C}$ & $\begin{array}{c}\text { SMP } \\
\text { exclusive } \\
\text { asym. pivotal }\end{array}$ & $\begin{array}{c}\text { SMP } \\
\text { sym. pivotal }\end{array}$ \\
\hline $\bar{Q}=180$ & $\mathrm{C}$ & $\begin{array}{c}\text { SMP } \\
\text { asym. pivotal }\end{array}$ & $\begin{array}{c}\text { SMP } \\
\text { asym. pivotal }\end{array}$ & \\
\hline
\end{tabular}

SMP: Structural market power; C: Competitive. The last cell is empty because $n k=120<180$ as an artifact of the experimental design.

\subsection{Full information}

Section 2.3.1 derives the linear-symmetric Supply Function Equilibria (SFE) for competitive situations with full information. Section 2.3.2 considers situations with structural market power.

\subsubsection{Symmetric SFE in competitive cases}

Consider a competitive situation where $n \geq 2$ symmetric bidders, each with a capacity $k>0$, can jointly serve the entire market at a constant marginal cost $c \in\left(0, P_{\max }\right)$. Demand and all technologies are common knowledge. As these bidders play a symmetric game, we concentrate the equilibrium analysis on symmetric equilibria. ${ }^{19}$

\footnotetext{
wind), which produces up to $\bar{q}$ at low marginal costs.

${ }^{18}$ One reason for why we chose $n=3$ is that this is between competitive and collusive in oligopolistic markets (Huck et al., 2004), which is a type of tension we do not want to rule out.

${ }^{19}$ This is the generic equilibrium type in such games (Hefti, 2017). There can be asymmetric SFE as well, but any asymmetric equilibrium is subject to a "coordination dilemma" as any permutation across the players again is an asymmetric equilibrium.
} 
In an SFE every bidder independently and non-cooperatively chooses her supply function $q_{j}$ to maximize her profit, taking the market situation $(s, \bar{Q})$ and the others' supply functions as given. To be precise, let $\mathcal{F}$ denote the set of all continuous supply functions $q:\left[0, P_{\max }\right] \rightarrow[0, k]$ with $q^{\prime}(P)>0$ whenever $q(P)>0$, and $q^{\prime \prime}(P) \geq 0$ whenever $q(P)=\bar{Q} / n .^{20}$ We first note that if $n k \geq \bar{Q}$ and each $q_{j} \in \mathcal{F}$, then any SFE satisfies $\sum_{j} q_{j}\left(P^{*}\right)=\bar{Q}$.

Lemma 1 Any SFE satisfies $\sum_{j} q_{j}\left(P^{*}\right)=\bar{Q}$, and $q_{j}\left(P^{*}\right)=\bar{Q} / n, \forall j$, in any symmetric SFE.

Definition 2 (SymSFE) A symmetric SFE (SymSFE) is a price $P^{*} \in\left[0, P_{\max }\right]$ and a profile of supply functions $\left(q_{j}\right)_{j=1}^{n}$ such that $q_{j}=q, j=1, \ldots, n, q \in \mathcal{F}, q\left(P^{*}\right)=\bar{Q} / n$, and

$$
q\left(P^{*}\right)\left(P^{*}-c\right) \geq q_{j}(P)(P-c)
$$

for any $q_{j} \in \mathcal{F}$ with $q_{j}(P)+(n-1) q(P)=\bar{Q}$.

Any SymSFE is conveniently summarized by $\left(P^{*}, q(\cdot)\right)$. We are particularly interested in the case where $q(P)$ is an affine-linear function on the relevant price segment.

Definition 3 (LinSFE) An affine-linear symmetric SFE (LinSFE) is a SymSFE with the property that $q(P)=\max \{\alpha+\beta P, 0\}$, where $\alpha \in \mathbb{R}$ and $\beta \geq 0$.

The parameter $\beta$ captures the price sensitivity of bids, while $\alpha$ corresponds to the base-load of a bid. To find a SymSFE, we can fix an arbitrary bidder $j$ and assume that all other $m \equiv n-1$ bidders choose an identical $\bar{q}(P) \in \mathcal{F}$. Firm $j$ 's payoff then is $\Pi_{j}(P)=(\bar{Q}-m \bar{q}(P))(P-c)$. The optimal strategy of firm $j$ is to choose $q_{j}(P) \in \mathcal{F}$ such that $\Pi_{j}(P)$ is maximized. Because $\bar{q}(P) \in \mathcal{F}$ a necessary and sufficient condition for $\left(P^{*}, q(\cdot)\right)$ to be a SymSFE is that ${ }^{21}$

$$
\bar{Q}-m q\left(P^{*}\right)-\left(P^{*}-c\right) m q^{\prime}\left(P^{*}\right)=0, \quad q\left(P^{*}\right)=\frac{\bar{Q}}{m+1} .
$$

The following theorem characterizes SymSFE and LinSFE.

Theorem 1 Let $m \equiv n-1 \geq 1$ denote the number of symmetric competitors of any bidder. Suppose that $k>\frac{\bar{Q}}{m}$, i.e., no single bidder is pivotal.

a) A price can be supported as a SymSFE if and only if $P \in\left(c, P_{\max }\right]$. For any given $P^{*} \in$ $\left(c, P_{\max }\right]$ the slope $q^{\prime}(P)$ is uniquely determined at $P^{*}$ for any supporting supply function

\footnotetext{
${ }^{20}$ The last qualification assures local convexity of $q(P)$ at symmetric equilibrium candidates, which helps to establish equilibrium existence.

${ }^{21}$ Also see Klemperer and Meyer (1989), section 2.
} 
profile. Moreover

$$
q^{\prime}\left(P_{0}^{*}\right)>q^{\prime}\left(P_{1}^{*}\right) \Leftrightarrow P_{0}^{*}<P_{1}^{*} \Leftrightarrow \Pi\left(P_{0}^{*}\right)<\Pi\left(P_{1}^{*}\right)
$$

b) Any price $P \in\left(c, P_{\max }\right]$ can be supported as a LinSFE, where the equilibrium supply function parameters $\left(\alpha^{*}, \beta^{*}\right)$ verify $\beta^{*}>0$ and

$$
\alpha^{*}+\beta^{*} c=\frac{m-1}{m(m+1)} \bar{Q} .
$$

While any feasible price above marginal costs can be a SymSFE, ${ }^{22}$ each price is characterized by a unique equilibrium slope of the supporting supply function. In other words, any equilibrium price has a unique linear supply function that implements it. The main implication is that LinSFE constitute the best linear approximation of the supply function in any SymSFE at the corresponding equilibrium price.

Corollary 1 If $\left(P^{*}, q(\cdot)\right)$ is a SymSFE, then the LinSFE $q(P)=\alpha^{*}+\beta^{*} P$ with

$$
\beta^{*}=\frac{\bar{Q}}{m(m+1)\left(P^{*}-c\right)}>0
$$

and $\alpha^{*}$ determined by $(3)$ is the best linear approximation to $q(\cdot)$ at $P^{*}$. Moreover, in the LinSFE with $\left(\alpha^{*}, \beta^{*}\right)$ as well as in any SymSFE $\left(P^{*}, q(P)\right)$ equilibrium profits are

$$
\Pi^{*}=\frac{\bar{Q}^{2}}{m(m+1)^{2} q^{\prime}\left(P^{*}\right)}>0 .
$$

where $q^{\prime}\left(P^{*}\right)=\beta^{*}$.

The left panel of Figure 1 illustrates two SymSFE together with their linear approximations (grey lines), which themselves constitute the two unique LinSFE for the prices $P_{0}^{*}, P_{1}^{*}$. These can be constructed by connecting point $A$ with point $B$ or $B^{\prime}$, and the slope $\beta\left(P^{*}\right)$ then corresponds to the slope $q^{\prime}\left(P^{*}\right)$ of any SymSFE with equilibrium price $P^{*}$. A central observation in Theorem 1 is that any LinSFE has to obey a unique equilibrium restriction (3). In Figure 1 this is vindicated by the fact that any two LinSFE intersect in the point A. We find a similar constraint in case of demand or supply uncertainty, and later utilize these observations to obtain a structural estimate of the average equilibrium bidding function.

\footnotetext{
${ }^{22}$ The multiplicity of SymSFE, ranging from quasi-competitive to collusive, reflects the general coordination problem in supply function competition (Klemperer and Meyer, 1989). Uniqueness requires additional assumptions, e.g., on the intercept $\alpha^{*}$. Green (1996) analyzed linear SFE with heterogeneous bidders and demand uncertainty under the assumption that each intercept was zero. Note from (3) that such a restriction annihilates the existence of a LinSFE in case of $m=1$.
} 

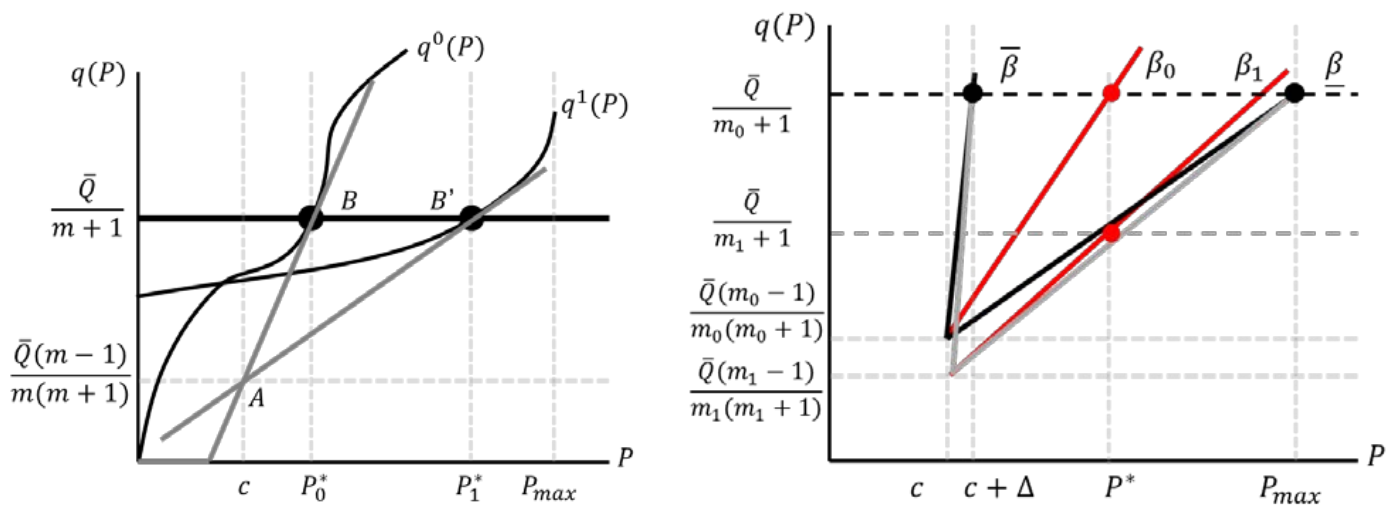

LEFT: Two different SymSFE $q^{0}(P), q^{1}(P)$ with their two unique supporting LinSFE.

RIGHT: The black and gray lines depict the range of all LinSFE for two different levels of competition $\left(m_{1}>m_{0} \geq 1\right)$ using the bounds in (6). Red lines: Obtaining the price $P^{*}$ under $m_{1}$ requires more bid shading than under $m_{0}\left(\beta_{1}<\beta_{0}\right)$.

Figure 1: SymSFE and LinSFE

It directly follows from $(4)$ that $\underline{\beta}=\frac{\bar{Q}}{m(m+1)(\bar{P}-c)}$ constitutes the lower bound of all admissible values of $\beta^{*}$ in a LinSFE. If prices increase in discrete steps of $\Delta>0$, as is the case in the experiment, Theorem 1 suggests $P=c+\Delta$ as the most competitive price in a LinSFE. By (4) this yields an upper bound $\bar{\beta}$ for $\beta^{*}$, and hence

$$
\beta^{*} \in[\underline{\beta}, \bar{\beta}]=\left[\frac{\bar{Q}}{m(m+1)(\bar{P}-c)}, \frac{\bar{Q}}{m(m+1) \Delta}\right]
$$

The range of all LinSFE implied by (6) is illustrated in the right panel of Figure 1 for two different market situations (black lines and gray lines, respectively).

Behavioral Market Power Comparing the various LinSFE identified by Theorem 1 and Corollary 2 shows that these are Pareto-ranked for the firms, and coordination on a higher price requires more bid shading (a lower slope coefficient $\beta$ ). Further, all LinSFE feature market power in the sense of Holt, stating that a Nash equilibrium features market power whenever it yields supracompetitive prices (Holt, 1989). This motivates the following minor qualification of Holt's definition in presence of multiple equilibria with behavioral market power (meaning that $P^{*}>c$ ).

Definition 4 (Behavioral market power ordering) Given two $\operatorname{LinSFE}\left(P_{0}, q_{0}(\cdot)\right)$ and $\left(P_{1}, q_{1}(\cdot)\right)$, and a fixed market situation $(s, \bar{Q}),\left(P_{1}, q_{1}(\cdot)\right)$ features more behavioral market power than $\left(P_{0}, q_{0}(\cdot)\right)$ if $P_{1}>P_{0}$.

The difference between behavioral market power and structural market power is that the former is defined in terms of an equilibrium outcome. Hence behavioral market power matters even if there is no structural market power. As we shall see below, pivotal market power results in SFE that may vary according to their behavioral market power as well. 
In competitive situations, it is likely that exogenous bidder characteristics and certain structural factors influence how much behavioral market power is exerted. In the empirical part, we control for the former by allowing for group-specific effects in the relevant estimations. Regarding structural factors, Figure 1 (right) illustrates how the set of LinSFE changes if the number of competitors $m$ varies. While the range of equilibrium prices itself is invariant to $m \geq 1$, coordination on higher prices requires more bid shading. ${ }^{23}$ In contrast, an increase in $\bar{Q}$ allows to support a fixed equilibrium price as a LinSFE with less individual bid shading. The following theorem makes these observations explicit. ${ }^{24}$

Corollary 2 (Competition and demand effects) A decrease in competition from $m$ to $m^{\prime} \geq$ 1 or an increase in demand $(d \bar{Q}>0)$ leads to more behavioral market power in the respective LinSFE's if and only if $\frac{\beta}{\beta^{\prime}}>\frac{m^{\prime}\left(m^{\prime}+1\right)}{m(m+1)}$, or $\frac{d \bar{Q}}{Q}>\frac{d \beta^{*}}{\beta^{*}}$, respectively.

The idea of Corollary 2 is to compare two specific LinSFE, characterized by $\beta$ and $\beta^{\prime}$, for two different market situations, holding either $\bar{Q}$ or $m$ fixed. The corollary then states precise conditions for how $\beta$ and $\beta^{\prime}$ must differ such that a decrease in competition or an increase in demand result in a higher equilibrium price. ${ }^{25}$ The intuition for the competition effect in Corollary 2 is that as long as the mere presence of more competitors does not make individual bidders overly optimistic about the possibility to collude at a high price, an increase in competition leads to individually more aggressive bidding. Likewise, an increase in $\bar{Q}$, meaning that "there is more for everyone", leads to higher prices as long as individual bidders do not overly reduce their bid shading. The constraint regression framework will allow us to empirically test whether changes in $m$ or $\bar{Q}$ affect the average $\beta$ 's in a way consistent with Corollary 2.

\subsubsection{SFE with structural market power}

Exclusive market power In case of exclusive market power with full information, Hefti and Shen (2019) establish that in market situation such as $\left(s_{3}, 60\right)$ there is a SFE, where the bidder with exclusive market power seizes the entire market at a clearing price equal to the second-lowest marginal costs. This parallels a known result in a Bertrand market featuring at least two firms with different marginal costs (Blume, 2003). By contrast, capturing the entire market is not necessarily attractive to the low-cost bidder with non-constant marginal costs, despite exclusive market power.

\footnotetext{
${ }^{23}$ Note that heuristic equilibrium selection, such as the midpoint method or the bidder-favoring equilibria as proposed by Brandts et al. (2014), would predict that the same price results independent of $m$ and $\bar{Q}$ in the current context.

${ }^{24}$ We omit the obvious proof.

${ }^{25}$ Note that, conversely, a lower equilibrium price does not generally assure a reduction in $\beta$; in this sense a lower price is necessary but not sufficient to conclude that bidding has become more competitive.
} 
As we show below, it may be better for such a bidder to share the market with high-cost firms to avoid the higher costs required by fully capturing the market.

Pivotal market power We first consider the case, where each of $n$ symmetric bidders is pivotal, meaning that $(n-1) \bar{k}<\bar{Q} \leq n \bar{k}$. In the experiment, this applies to market situation $\left(s_{4}, 100\right)$.

Proposition 1 (Symmetric pivotal market power) Consider the case of $n>1$ symmetric bidders with $c>0$, and $(n-1) \bar{k}<\bar{Q} \leq n \bar{k}$. There is $\hat{P} \in\left(c, P_{\max }\right)$ such that no SFE exists with $P^{*}<\hat{P}$. Further, any $P \in\left[\hat{P}, P_{\max }\right]$ can be supported as a LinSFE, where $\left(\alpha^{*}, \beta^{*}\right), P^{*}$ and $\Pi^{*}$ are described by (3), (4) and (5), respectively.

The intuition for the lower price bound $\hat{P}$ is that pivotal market power works like an outside option for any given bidder. Because of pivotality, a bidder can always shade her bids up to the price cap and still supply a positive quantity. Hence any SFE must yield at least the same profit in order to make such a deviation unprofitable. As illustrated in Figure 2 this implies that a certain range of competitive prices cannot be supported as SFE compared to the situation where capacities are such that no firm is pivotal. ${ }^{26}$ In this sense, pivotal market power can be thought of as increasing the behavioral market power of the bidders.

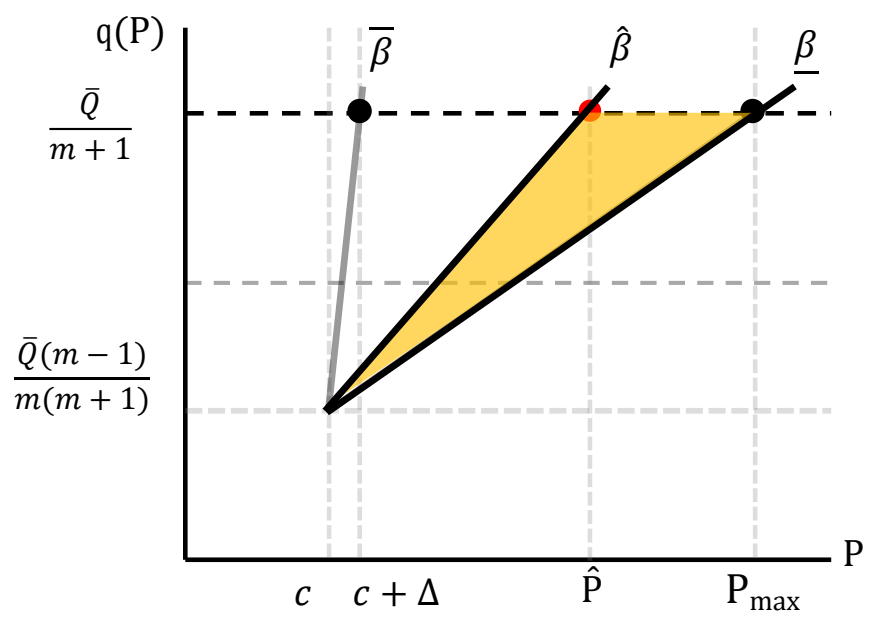

The figure shows the equilibrium range of LinSFE without pivotal market power $(\underline{\beta}, \bar{\beta})$ and with pivotal power $(\hat{\beta}, \bar{\beta})$.

Figure 2: Symmetric pivotal market power

We next consider asymmetric pivotal market power. In the experiment, situations $\left(s_{3}, 100\right)$ and $\left(s_{3}, 180\right)$ both feature asymmetric pivotal market power. The two situations further differ in thus that the low-cost firm also has exclusive market power in the former but not in the latter.

\footnotetext{
${ }^{26}$ In case of demand uncertain, Brandts et al. (2014) show that symmetric pivotal market power reduces the range of smooth SFE in a similar way.
} 
Proposition 2 (Asymmetric pivotal power) In situation $\left(s_{3}, 180\right)$ no price below $P=91.67$ can result in an SFE. With price steps of $\Delta=5$, this suggests $P^{*}=100$ as equilibrium outcome.

The lower bound of $P=91.67$ follows alone from pivotality, as the low-cost bidder can always assure herself at least a quantity of $q=100$ at the price cap.

We now turn to situation $\left(s_{3}, 100\right)$, which jointly features exclusive and (asymmetric) pivotal market power of the low-cost bidder. In contrast to situation $\left(s_{3}, 60\right)$, seizing the market now involves increasing marginal costs for the low-cost bidder. The parameters of the experiment were chosen such that fully exploiting the pivotal market power is not optimal for the single low-cost bidder, provided that the high-cost bidders supply enough quantity at prices below $P_{\max }=100$. This contrasts with the maximal bid shading of the low-cost bidder in situation $\left(s_{3}, 180\right)$ identified by Proposition 2. However, it is also not optimal for the low-cost bidder to capture the market at $P=\bar{c}$, unless high-cost bidders supply a lot of their quantity at their marginal costs (which is not profitable). The intuition is that the last units required by market capturing at $P=\bar{c}$ are not profitable due to the increased marginal costs. By contrast, if the low-cost bidder shades her bids to reach a price above $\bar{c}$ this allows her to sell at least her lower-cost units at a higher price. The following proposition shows, for a discrete price space with price steps of $\Delta=5$ as in the experiment, that the first price above high marginal costs, $P^{*}=\bar{c}+\Delta=55$, is part of a SFE once high-cost bidders supply enough quantity at $P=55$.

Proposition 3 Let $\bar{c}=50$, and $j=2,3$ indicate the two high-cost bidders in situation $\left(s_{3}, 100\right)$. Any supply function $q_{L}(P)$ of the single low-cost bidder with $q_{L}(\bar{c})=80$ and $q_{L}(P)=120$ for $P>\bar{c}$ together with any high-cost supply function profile $\left(q_{2}, q_{3}\right)$ such that $\sum_{j \geq 2} q_{j}(\bar{c})<20$ and $q_{j}(P)=40$ for $j=2,3$ and $P>\bar{c}$ is a SFE with $P^{*}=55$ and $q_{L}^{*}=80$.

\subsection{SFE with uncertainty}

We now analyze the SFE in the two information treatments where bidders have limited information, which requires them to form expectations about the possible scenarios.

\subsubsection{Supply uncertainty: Averaged linear symmetric SFE}

In this section, we consider the case where each bidder knows her own technology and the market demand, but not the technologies of the other bidders. Consider a bidder $j$ with marginal costs $c>0$, who is unsure about how many competitors are present in the market. Specifically, let $m \in\{0,1, \ldots, M\}$ be the number of symmetric competitors $(M \equiv n-1 \geq 1)$ of a firm, where $m$ is distributed according to $F(\cdot)$ with mean $\bar{m}$. In our experiment, the relevant market situations are 
those, where at least one low-cost bidder is present (states $s_{1}-s_{3}$ ). A low-cost bidder $j$ then may face $m \in\{0,1,2\}$ identical other bidders, while $2-m$ bidders have high costs. Hence $j$ is unsure about how much competition from low-cost bidders she faces on the price range $\left[0, P_{\max }\right)$, where $P_{\max }=\bar{c}+\Delta$ is the first price that is profitable to high-cost bidders.

If bidder $j$ assumes that each other symmetric bidder present in the market bids according to the same supply function $\bar{q}(P), j$ faces a (random) residual demand $\bar{Q}-m \bar{q}(P)$, and her expected payoff is

$$
E[\Pi]=E\left[\left(\bar{Q}-m \bar{q}\left(P_{m}\right)\right)\left(P_{m}-c\right)\right] .
$$

In a linear symmetric SFE, all bidders play the same supply function $q(P)=\alpha+\beta P$. In such an equilibrium, the state-wise market clearing conditions

$$
\alpha+\beta P_{m}=\frac{\bar{Q}}{m+1}, \quad m=0,1, \ldots, M
$$

are satisfied, which further implies that the market must also clear on average

$$
\alpha+\beta \bar{P}=\bar{Q} \mu, \quad \bar{P} \equiv E\left[P_{m}\right], \quad E\left[\frac{1}{m+1}\right] \equiv \mu .
$$

Bayesian players would take into account that the optimal price in (7), $P_{m}$, depends on $m$. Given $\bar{q}(P)$, a higher price then conveys the "good" news to bidder $j$ that there are not many symmetric competitors present in the market. Working through this logic reveals that globally affine-linear Bayesian SFE generally do not exist (see Appendix B.1). In the following, we show that an affine-linear SFE exists under the behavioral assumption that each bidder resolves the uncertainty by acting as if $\bar{m}$ other bidders were in the market for sure. Loosely spoken, this means that bidders play against the average market situation, rather than forming the precise beliefs about the number of symmetric bidders present in the market for different possible prices. If bidder $j$ believes to compete against $\bar{m}$ bidders, each of which chooses the same affine-linear supply function $\bar{q}(P)$, then $j$ faces the residual demand $\bar{Q}-\bar{m} \bar{q}(P)$. Accordingly, $j$ chooses her own supply function $q(P)$ such that $P$ maximizes

$$
\Pi(P)=(\bar{Q}-\bar{m}(\alpha+\beta P))(P-c) .
$$

By the first-order condition, the aspired price is

$$
P^{*}=\frac{\bar{Q}-\alpha \bar{m}+c \beta \bar{m}}{2 \beta \bar{m}} .
$$


A consistency requirement is that $P^{*}=\bar{P}=E\left[P_{m}^{*}\right]$, meaning that the true average auction price indeed corresponds to the aspired optimal price $P^{*}$ by each bidder. We define an averaged linear symmetric SFE (A-LinSFE) as a choice of identical affine-linear supply functions $q(P)=\alpha^{*}+\beta^{*} P$, $\beta^{*}>0$, such that i) $P^{*}$ in (11) maximizes (10), ii) market clearing (9) holds, and iii) the consistency condition $\bar{P}=P^{*}$ is verified. Thus, in an A-LinSFE each "active" bidder plays as if $\bar{m}$ identical bidders were present, and her payoff maximizing price $P^{*}$ corresponds to the expected auction price.

A useful observation is that all supply functions satisfying the requirements of A-LinSFE must also verify a linear constraint on $\left(\alpha^{*}, \beta^{*}\right)$ similar to full information.

Theorem 2 Let $\frac{\bar{m}}{m+1}>2 \mu \bar{m}-1$ for $m \in\{0,1, \ldots, M\}$. The parameters $\left(\alpha^{*}, \beta^{*}\right)$ constitute an $A$-LinSFE $q(P)=\alpha^{*}+\beta^{*} P$ with positive profit in each state $m=0,1, \ldots, M$ iff $\beta^{*}>0$ and

$$
\begin{gathered}
\alpha+c \beta=\frac{\bar{Q}}{\bar{m}}(2 \mu \bar{m}-1), \\
\beta^{*} \geq \frac{\bar{Q}(1+\bar{m}(1-2 \mu))}{\bar{m}\left(P_{\max }-c\right)} .
\end{gathered}
$$

The average price is

$$
P^{*}=c+\frac{\bar{Q}(1-\mu \bar{m})}{\bar{m} \beta^{*}}>c,
$$

any any average price

$$
P^{*} \in\left(c, c+\frac{\left(P_{\max }-c\right)(1-\bar{m} \mu)}{1+\bar{m}(1-2 \mu)}\right]
$$

can be supported as A-LinSFE. The state-specific prices $P_{m}^{*}>c$ are given by

$$
P_{m}^{*}=c+\frac{\bar{Q}}{\beta} \frac{\bar{m}-(m+1)(2 \mu \bar{m}-1)}{\bar{m}(m+1)} .
$$

The parametric requirement at the outset of Theorem 2 implies that $P_{m}^{*}>c$ in any A-LinSFE, meaning that each bidder obtains a strictly positive profit in any state $m .^{27}$ An important implication of Theorem 2 is that the highest possible state-wise price, $P_{m}^{\max }$, attainable in an A-LinSFE is strictly below $P_{\max }$ whenever $m>0$.

Corollary 3 In any A-LinSFE, the state-wise price $P_{m}^{*}$ is strictly decreasing in $m$, and $P_{m}^{*}$ and $P^{*}$ both increase in $\bar{Q}$ if and only if $\frac{d \bar{Q}}{\bar{Q}}>\frac{d \beta^{*}}{\beta^{*}}$. The A-LinSFE featuring most behavioral market power verifies $P_{0}^{*}=P_{\max }$, and $P_{m}^{*}<P_{\max }$ whenever $m>0$.

\footnotetext{
${ }^{27}$ The condition holds with the parameters of our experiment, where $M=2$ and $\bar{m}=1 / 4 * 0+1 / 2 * 1+1 / 4 * 2=1$ as well as $\mu=1 / 4 * 1+1 / 2 * 1 / 2+1 / 4 * 1 / 3=7 / 12$.
} 
Figure 3 illustrates the range of possible A-LinSFE for the case where $M=2$ as in the experiment. The figure uses

$$
\beta^{*} \in[\underline{\beta}, \bar{\beta}]=\left[\frac{\bar{Q}(1+\bar{m}(1-2 \mu))}{\bar{m}\left(P_{\max }-c\right)}, \frac{\bar{Q}((1+M)(1-2 \bar{m} \mu)+\bar{m})}{(1+M) \bar{m} \Delta}\right]
$$

as the range of admissible $\beta^{*}$. The upper bound $\bar{\beta}$ is such that $P_{M}^{*}=c+\Delta$, i.e., the lowest

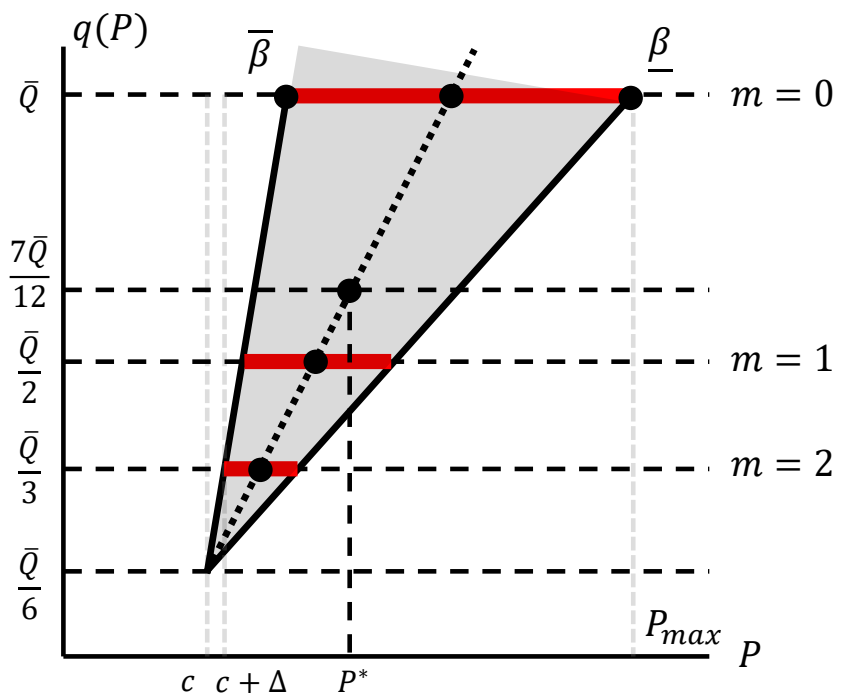

The figure shows the equilibrium range of A-LinSFE if $M=2$ (shaded area) and the state-wise equilibrium range of prices (red lines). The dotted line illustrates a particular A-LinSFE (midpoint equilibrium).

Figure 3: A-LinSFE with supply uncertainty

price in the most competitive A-LinSFE is the first price just above $c$. Similar to full information, any $P^{*}$ that is supported as A-LinSFE has a uniquely defined supply function $q(P)=\alpha^{*}+\beta^{*} P$ associated with it. Moreover, the state-wise prices $P_{m}^{*}$ must reside on the same equilibrium supply function in any given A-LinSFE, as illustrated in Figure 3 (dotted line). This simple property can be empirically assessed with the bidding data of our experiment.

The central result in Corollary 3 is that there cannot be an A-LinSFE with $P_{m}^{*}=P_{\max }$ for some $m>0$. The reason is that such an equilibrium would yield an average market price $\bar{P}$ above the aspired equilibrium price $P^{*}$, contradicting the consistency condition that $\bar{P}=P^{*}$. Further, $P_{m}^{*}$ always decrease strictly in $m$ in any given A-LinSFE, because more identically behaving competitors lead to a larger aggregate supply, which yields a strictly lower auction price. Combining these two facts shows that the full information prices featuring high behavioral market power, with prices close to or equal $P_{\max }$ despite $m>0$, are not attainable as outcomes of A-LinSFE. In Figure 3 , this is illustrated with red lines, depicting all prices that are supported by A-LinSFE. Except for $m=0$, this is only a lower subset of the prices attainable as LinSFE under full information $\left(\left[c+\Delta, P_{\max }\right], \forall m\right)$.

This simple analysis suggests that "playing against the average", as embodied in the notion of 
A-LinSFE, tends to make bidders pessimistic about high prices. The main empirical conjecture we form based on this is that with supply uncertainty the auction price averaged across all states $m=0, \ldots, M$ should, at the very least, not exceed its full information counterpart. Indeed, it is immediate to verify that heuristic equilibrium selection rules, such as the "midpoint equilibrium" (dottet line in Figure 3) or the "bidder-preferred equilibrium" (Brandts et al., 2014), yield a strictly lower average price in case of supply uncertainty with A-LinSFE as the solution concept, compared to LinSFE in full information. ${ }^{28}$

Finally, we note that equilibrium prices $P_{m}^{*}, m>0$, and $P^{*}$ increase in $\bar{Q}$ under the same condition on $\beta^{*}$ as in Corollary 2. By contrast, $P_{m}^{*}$ always decreases in $m$ in any given A-LinSFE, while we need an additional requirement on how $\beta$ depends on $m$ under full information to assure a similar conclusion, because bidding then always is contingent on the number of competitors.

\subsubsection{Demand uncertainty: Averaged linear symmetric SFE}

We now turn to the case, where each of $n>1$ identical bidders knows the number $m=n-1$ of competitors in the market and all technologies, but not the demand level. Such demand uncertainty is a leading assumption in most of the literature on SFE. Let $\left\{\bar{Q}_{1}, \ldots, \bar{Q}_{v}\right\}$ be the possible demand states, where $0<\bar{Q}_{1}<, \ldots,<\bar{Q}_{v}$ and $\Gamma \equiv E(\bar{Q})>0$. We analyze the auction by the concept of A-LinSFE. Suppose that a bidder faces $m>0$ symmetric competitors, each of which bids according to $\bar{q}(P)=\alpha+\beta P$. The bidder seeks to find the price $P^{*}$ that maximizes

$$
\Pi(P)=(\Gamma-m \bar{q}(P))(P-c)
$$

the first-order condition for the optimal price being

$$
P^{*}=\frac{\Gamma-\alpha m+c \beta m}{2 \beta m} .
$$

The state-wise and average market clearing conditions in a symmetric equilibrium are

$$
\begin{gathered}
\alpha+\beta P_{\bar{Q}}=\frac{\bar{Q}}{m+1}, \quad \bar{Q}=\bar{Q}_{1}, \ldots, \bar{Q}_{v} \\
\alpha+\beta \bar{P}=\frac{\Gamma}{m+1}, \quad \bar{P} \equiv E\left[P_{\bar{Q}}\right] .
\end{gathered}
$$

\footnotetext{
${ }^{28}$ It is a theoretical possibility that the average price under full information is lower than the one with supply uncertainty. This could happen, e.g., if low-cost bidders coordinate on the most preferred A-LinSFE under supply uncertainty, but play the most competitive LinSFE in cases $m>0$ under full information. However, these two outcomes seem contradictory. The second states that bidders respond aggressively to a competitive threat, which means that they also should do so under supply uncertainty, as a competitive threat exists at least on expectation.
} 
Similar to section 2.4.1 we define an A-LinSFE with demand uncertainty as a selection of identical, increasing supply functions $q(P)=\alpha^{*}+\beta^{*} P$, such that i) $P^{*}$ in (19) maximizes (18), ii) market clearing (21) holds, and iii) $\bar{P}=P^{*}$. The following theorem characterizes the A-LinSFE with demand uncertainty given that there is no pivotal market power on average $(m k>\Gamma)$.

Theorem 3 Let $m>0$ and capacities be such that $m k>\Gamma$, and $m \bar{Q}_{1}-(m-1) \Gamma>0$. The parameters $\left(\alpha^{*}, \beta^{*}\right)$ are a A-LinSFE $q(P)=\alpha^{*}+\beta^{*} P$ with positive profit in each state $\bar{Q}=$ $\bar{Q}_{1}, \ldots, \bar{Q}_{v}$ if and only if $\beta^{*}>0$ and

$$
\begin{gathered}
\alpha^{*}+c \beta^{*}=\frac{m-1}{m(m+1)} \Gamma, \\
\beta^{*} \geq \frac{m \bar{Q}_{v}-(m-1) \Gamma}{m(1+m)\left(P_{\max }-c\right)} .
\end{gathered}
$$

The average price is

$$
P^{*}=c+\frac{\Gamma}{m(m+1) \beta^{*}},
$$

and any average price

$$
P^{*} \in\left(c, c+\frac{\Gamma\left(P_{\max }-c\right)}{m \bar{Q}_{v}-(m-1) \Gamma}\right]
$$

can be supported as an $A$-LinSFE, and the state-specific prices $P_{\bar{Q}}^{*}$ for $\bar{Q} \in\left\{\bar{Q}_{1}, \ldots, \bar{Q}_{v}\right\}$ are

$$
P_{\bar{Q}}^{*}=c+\frac{m \bar{Q}-(m-1) \Gamma}{\beta^{*} m(m+1)}>c .
$$

The A-LinSFE in Theorem 3 have similar properties as those from supply uncertainty.

Corollary 4 In any A-LinSFE, the state-wise price $P_{\bar{Q}}^{*}$ is strictly increasing in $\bar{Q}$, and if $\beta^{\prime}(m) \geq$ 0 , then $P^{*}$ decreases in $m$. If additionally $\Gamma(m(m-2)-1)<m^{2} \bar{Q}$, then $P_{\bar{Q}}^{*}$ decreases in $m$. The A-LinSFE featuring most behavioral market power verifies $P_{\bar{Q}}^{*}=P_{\max }$, and $P_{\bar{Q}}^{*}<P_{\max }$ for any $\bar{Q}<\bar{Q}_{v}$.

Like with supply uncertainty, demand uncertainty limits the behavioral market power exerted in A-LinSFE compared to the full information case, because bidders are forced to average across different market situations. Consequently, the state-wise collusive equilibria are not attainable as (state-wise) outcomes of A-LinSFE. Accordingly, our main conjecture is that demand uncertainty does not lead to higher prices, averaged across demand levels, compared to the full information counterpart. Further, the facts that aggregate supply in any A-LinSFE is strictly increasing implies that a realization of $\bar{Q}$ must automatically lead to a higher price. This contrasts with the full information case (Corollary 2 and Corollary 4), where an additional condition on $\beta$ is required for 
the same result. Finally, if $c+\Delta$ is the first price above $c$, (23) suggests

$$
\beta^{*} \in[\underline{\beta}, \bar{\beta}]=\left[\frac{m \bar{Q}_{v}-(m-1) \Gamma}{m(1+m)\left(P_{\max }-c\right)}, \frac{m \bar{Q}_{1}-(m-1) \Gamma}{m(1+m) \Delta}\right] .
$$

as the range of admissible $\beta^{*}$. The upper bound $\bar{\beta}$ is such that $P_{\bar{Q}_{1}}^{*}=c+\Delta$, i.e., the lowest price in the most competitive A-LinSFE is the first price just above $c$.

The A-LinSFE identified by Theorem 3 show certain similarities to the symmetric Bayesian SFE in the case where demand uncertainty takes on the form of a continuous random variable with convex support, as in Klemperer and Meyer (1989). Then, the symmetric Bayesian SFE with constant marginal costs are described by a set of differential equations, the general solution of which has the form $q(P)=\gamma(P-c)^{m}$, where $\gamma>0$ is a constant (see, e.g., Holmberg, 2008). In the special case where $m=1$, it follows from (22) and $\beta^{*}>0$ that the functional forms of A-LinSFE and the symmetric Bayesian SFE coincide. Further, Klemperer and Meyer (1989) and Holmberg (2008) find that the market-clearing price must fall if the number of identical competitors is increased. By Corollary 4, this result holds under reasonable conditions (the presence of more competitors does not make individual bidders behave less competitively) in A-LinSFE as well.

Finally, given a fixed convex support of demand, the distribution of demand itself has no effect at all on the symmetric Bayesian SFE in a symmetric constant marginal cost setting. Bolle et al. (2013) test this hypothesis experimentally, and find significant differences in the average supply functions if the demand distribution is varied on a fixed support. Specifically, the three empirical demand distributions implemented in that paper yield different means $\Gamma$, and the average empirical bidding becomes less competitive in treatments with higher $\Gamma$, contradicting the Bayesian SFE prediction. In contrast, the average demand $\Gamma$ may affect equilibrium bidding in A-LinSFE whenever $m>1$ by (22). As with full information, an increase in (expected) demand $\Gamma$ makes it rather unlikely that bidders coordinate on an equilibrium involving less bid shading (higher $\beta^{*}$ ). Indeed, (24) shows that $P^{*}$ increases in $\Gamma$ iff $\frac{d \Gamma}{\Gamma}>\frac{d \beta^{*}}{\beta^{*}}$. In this sense, the finding by Bolle et al. (2013) appears reasonable from the perspective of A-LinSFE.

Demand uncertainty may limit the extent of strategic bid shading due to pivotal market power. To see this in the specific context of the experiment, note that in case of only symmetric bidders with high costs (state $s_{4}$ ), there is no pivotal market power on expectation, ${ }^{29}$ while situation $\left(s_{4}, 100\right)$ entails symmetric pivotal market power with full information. We therefore conjecture

\footnotetext{
${ }^{29}$ As a consequence of the experimental design, each bidder can sell her full capacity at the maximal price in situation $\left(s_{4}, 180\right)$. Given $s_{4}$, therefore only the case $\bar{Q}=60,100$ matters for strategic bidding, where each demand level occurs with equal probability.
} 
that equilibrium prices in situation $\left(s_{4}, 100\right)$ are lower with demand uncertainty than with full information. In contrast, an advantaged bidder may still exert substantial market power despite demand uncertainty. As the next proposition exemplifies, such a bidder may shade bids in a way that allows her to fully exploit her market power despite demand uncertainty.

Proposition 4 Consider state $s_{3}$ of the experimental setup with demand uncertainty. The following supply functions then constitute a SFE. Each high-cost bidder sets $q_{H}(P)=40$ for $P>\bar{c}$ and $q_{H}(P)=0$ else. The low-cost bidder sets

$$
q_{L}(P) \begin{cases}<60, & P<\bar{c} \\ =80, & P_{\max }>P \geq \bar{c} \\ =120, & P=P_{\max } .\end{cases}
$$

The resulting equilibrium prices for the various possible demand levels are $P_{60}^{*}=\bar{c}=50, P_{100}^{*}=$ $\bar{c}+\Delta=55$ and $P_{180}^{*}=P_{\max }=100$.

Recall from the analysis under full information that in state $s_{3}$ the single low-cost bidder wishes to exploit either her exclusive or her pivotal market power, depending on the demand level. By Proposition 4 this is still possible, despite demand uncertainty, as the low-cost bidder can effectively balance the partial bid shading required by market capturing with the pivotal price cap strategy. Key for the equilibrium in Proposition 4 is that the bidder can submit an entire function to the market, which allows her to effectively shade bids, and secure demand, over the entire price range.

\subsection{Empirical hypothesis}

Information effects and market power Our primary interests is the comparison between auction outcome and strategic bidding under the various information treatments. We now summarize the main observations from sections 2.3-2.4 as testable empirical hypotheses below, starting with the case of pivotal market power under full information.

\section{H1: Pivotal market power with full information}

In situation $\left(s_{4}, 100\right)$ the average price is $P \in[80,100]$, in situation $\left(s_{3}, 180\right)$ the average price is $P=100$, and in situation $\left(s_{3}, 100\right)$ the average price verifies $P=55$.

Hypothesis $\mathrm{H} 1$ refers to the two situations without exclusive but either with symmetric $\left(s_{4}, 100\right)$ or asymmetric $\left(s_{3}, 180\right)$ pivotal power, and to the mixed situation $\left(s_{3}, 100\right)$, where both pivotal and exclusive market power coexist. In $\left(s_{4}, 100\right)$ and $\left(s_{3}, 180\right)$ we expect to observe high prices as a consequence of strategic bid shading due to pivotal market power (Propositions 1 and 2). ${ }^{30}$

\footnotetext{
${ }^{30}$ Situation $\left(s_{2}, 180\right)$ also features symmetric pivotal market power in such that the two low-cost bidders can jointly
} 
In situation $\left(s_{3}, 100\right)$, it is optimal for the low-cost bidder to share the market with her high-cost rivals (Proposition 3), leading to a price $P^{*}=\bar{c}+\Delta=55$.

The comparison between full information (Theorem 1, Corollary 1) with cost and demand uncertainty (Corollaries 3 and 4) suggests that limited information should not lead to higher equilibrium prices on average if the (A)-LinSFE approach provide an appropriate description of the average bidding behavior. The reason is that if bidders internalize the respective uncertainty by averaging the various market situations, the range of average prices that can be supported as equilibrium outcomes is reduced. For example, if bidders coordinate on an intermediate price in all information treatments, the corresponding average price in each uncertainty treatments must be strictly lower than its full information counterpart. The specific predictions are as follows.

In case of supply uncertainty, the theory in section 2.4.1 applies to market situations involving at least one low-cost bidder and a demand level $\bar{Q} \in\{60,100\}$, assuring that there is no pivotal market power on average. ${ }^{31}$ Our primary hypothesis is that the price averaged across all relevant market situations is (at least weakly) lower with supply uncertainty than with full information. In particular, supply uncertainty most likely leads to lower prices in the specific situations $\left(s_{3}, 60\right)$, $\left(s_{3}, 100\right)$. These situations feature a single low-cost bidder with exclusive market power and, by the analysis of Section 2.3.2, we should observe average prices around $P=\bar{c}=50$ with full information. By contrast, obtaining such a price in either situation with supply uncertainty requires bidders to coordinate on the A-LinSFE with maximal behavioral market power (Corollary 3). This is an unlikely outcome, provided that we do not observe a similar cooperative tendency with full information. We further expect supply uncertainty to break the pivotal market power of situation $\left(s_{4}, 100\right)$ with full information. It is conceivable that high-cost bidders play more aggressively under supply uncertainty to eventually seize some residual demand provided that the low-cost bidders, if present, fail to capture the market at $P \leq 50$. Then, we should observe prices close to $P=50$ if situation $\left(s_{4}, 100\right)$ occurs, where actually no low-cost bidder is present. In contrast, the pivotal market power in $\left(s_{4}, 100\right)$ under full information pushes prices above $P=80$ by Hypothesis H1.

\section{H2: Supply uncertainty effects}

H2a: Let $\bar{Q} \in\{60,100\}$. The price averaged over all market situations involving at least one low-cost bidder with supply uncertainty does not exceed its full information counterpart.

H2b: The average prices in situations $\left(s_{3}, 60\right),\left(s_{3}, 100\right)$ and $\left(s_{4}, 100\right)$ with supply uncertainty do not exceed their full information counterparts.

We now turn attention to demand uncertainty; the relevant theoretical underpinning is provided

serve the market. However, any such equilibrium involves that at least one bidder experiences increasing marginal costs which was not part of the equilibrium analysis.

${ }^{31}$ For $\bar{Q}=180$, a low-cost bidder has pivotal market power on average, as she can expect to compete with one other low-cost competitor on average. 
by Theorem 3 and Corollary 4.

\section{H3: Demand uncertainty effects}

H3a: In states $s_{2}$ or $s_{4}$ the price averaged across all demand states with demand uncertainty does not exceed its full information counterpart. ${ }^{32}$

H3b: The average price in situation $\left(s_{4}, 100\right)$ with demand uncertainty does not exceed its full information counterpart. In contrast, the average price in all states $\left(s_{3}, \bar{Q}\right), \bar{Q} \in\{60,100,180\}$, is the same with demand uncertainty and full information.

The basis for Hypothesis H3b are expression (26) and Proposition 4. The former implies a price range of $[65,100]$ by $(27)$ in situation $\left(s_{4}, 100\right)$ with demand uncertainty, which contrasts to the price interval $[80,100]$ predicted with full information (H1).

Competition and demand effects The second main prediction pertains to competition and demand effects in competitive situations as identified by Corollary 2 (full info) and Corollaries 3 , 4 (cost and demand uncertainty, respectively). The overarching prediction is that an increase in competition $(d m>0)$ or a decrease in demand $(d \bar{Q}<0)$ should lead to lower equilibrium prices.

\section{H4: Competition and demand effects}

Denote by $P(s, \bar{Q})$ the average price if the state is $s$ and demand is $\bar{Q}$.

H4a (Full information): $P\left(s_{1}, \bar{Q}\right)<P\left(s_{2}, \bar{Q}\right)$ for $\bar{Q} \in\{60,100\}, P(s, 60)<P(s, 100)$ for $s \in\left\{s_{1}, s_{2}, s_{4}\right\}$ and $P\left(s_{1}, 100\right)<P\left(s_{1}, 180\right)$.

H4b (Supply uncertainty): $P\left(s_{1}, \bar{Q}\right)<P\left(s_{2}, \bar{Q}\right)<P\left(s_{3}, \bar{Q}\right)$ for $\bar{Q} \in\{60,100\}$, and $P(s, 60)<$ $P(s, 100)$ for $s \in\left\{s_{1}, s_{2}, s_{3}\right\}$

H4c (Demand uncertainty): $P\left(s_{2}, 60\right)<P\left(s_{2}, 100\right)<P\left(s_{2}, 180\right)$ and $P\left(s_{4}, 60\right)<P\left(s_{4}, 100\right)$.

Supra-competitive equilibria The SFE analysis in competitive situations broadly predicts equilibrium prices to be above marginal costs, because marginal-cost pricing would require an infinitely steep supply function, which is not viable as LinSFE or A-LinSFE, respectively. ${ }^{33}$ In the experiment, the first price above marginal costs is $c+\Delta$, where $c \in\{\underline{\mathrm{c}}, \bar{c}\}$ depending on the market situation. The theory of LinSFE with full information developed in section 2.3.1 applies to states $s_{1}, s_{2}, s_{4}$ with $\bar{Q}=60,100$ in the experiment, and to $\left(s_{1}, 180\right) \cdot{ }^{34}$ Specifically, states $s_{1}, s_{4}$ feature $n=3$ symmetric bidders, either all with $c=10$ or $c=50$ in the relevant quantity range, and $P_{\max }=100$. State $s_{2}$ features two low-cost and one high-cost bidder, where for $\bar{Q} \in\{60,100\}$ the relevant marginal costs and price range are $c=10$ and $[0,50]$, respectively.

\footnotetext{
${ }^{32}$ State $s_{1}$ together with the parameters of the experiment violate condition (27).

${ }^{33}$ Also see Klemperer and Meyer (1989), who find that all SFE involve non-negative price-cost markups in case of demand uncertainty.

${ }^{34}$ In $\left(s_{2}, 180\right)$ a symmetric equilibrium would involve a quantity of $q=90>80$ for both low-cost bidders, which invalidates the assumption of constant marginal costs on the relevant range.
} 
With demand or supply uncertainty, bidders cannot condition their supply function on the exact market situation $(s, \bar{Q})$. In case of supply uncertainty, the theory from section 2.4 .1 applies to situations $(s, \bar{Q})$ with $s \in\left\{s_{1}, s_{2}, s_{3}\right\}$ and $\bar{Q} \in\{60,100\}$. In this respect, we can check whether the average empirical prices belong to the interval (15), which evaluates to $P^{*} \in[22.5,32.5] .{ }^{35}$ In case of demand uncertainty, the results in Theorem 3 apply to situations $\left(s_{4}, \bar{Q}\right)$ and $\left(s_{2}, \bar{Q}\right)$, and the relevant price interval $(25)$ evaluates to $P_{s_{2}}^{*} \in[19.44,38.33]$ and $P_{s_{4}}^{*} \in[60,83.33] .{ }^{36}$

In both uncertainty treatments, the supply functions in A-LinSFE impose additional structure on all state-wise prices, as they must lie on the same linear supply function. Two testable implications emerge. First, if the most competitive A-LinSFE is played, this yields the minimal prices of 15,20 and 35 in states $s_{1}-s_{3}$, respectively, with supply uncertainty. These numbers give a lower bound on average prices, and as such a simple consistency test for A-LinSFE. With demand uncertainty, similar lower bounds emerge. Evaluating Theorem 3 in situation $\left(s_{2}, \bar{Q}\right)$ shows that $P_{\bar{Q}}^{*} \geq 10+\frac{\bar{Q}}{12}$. In case of $s_{4}$, the relevant uncertainty between bidders reduces to $\bar{Q}=60,100$, where each happens with equal probability, and it follows that $P_{\bar{Q}}^{*} \geq 50+\frac{\bar{Q}-40}{4}$ in these cases. Second, we can verify whether the true state-wise average prices indeed lie on the A-LinSFE that empirically best matches the data.

\section{H5: Supra-competitive equilibria}

Average equilibrium prices satisfy the boundary requirements summarized in Table 2.

Table 2: Supra-competitive equilibria hypothesis

\begin{tabular}{|c|c|c|c|c|c|c|}
\hline & & $s_{1}$ & $s_{2}$ & $s_{3}$ & $s_{4}$ & Pooled $\left(s_{1}, s_{2}, s_{3}\right)$ \\
\hline \multirow{3}{*}{$\bar{Q}=60$} & $T_{B}$ & $\geq 15$ & $\geq 15$ & - & $\geq 55$ & - \\
\hline & $T_{D}$ & - & $\geq 15$ & - & $\geq 55$ & - \\
\hline & $T_{S}$ & $\geq 15$ & $\geq 20$ & $\geq 35$ & - & $\in[22.5,32.5]$ \\
\hline \multirow{3}{*}{$\bar{Q}=100$} & $T_{B}$ & $\geq 15$ & $\geq 15$ & - & $\geq 55$ & - \\
\hline & $T_{D}$ & - & $\geq 18.33$ & - & $\geq 65$ & - \\
\hline & $T_{S}$ & $\geq 15$ & $\geq 20$ & $\geq 35$ & - & $\in[22.5,32.5]$ \\
\hline \multirow{3}{*}{$\bar{Q}=180$} & $T_{B}$ & $\geq 15$ & - & - & - & - \\
\hline & $T_{D}$ & - & $\geq 25$ & - & - & - \\
\hline & $T_{S}$ & - & - & - & - & - \\
\hline \multirow{3}{*}{ Pooled } & $T_{B}$ & - & - & - & - & - \\
\hline & $T_{D}$ & - & $\in[19.44,38.33]$ & - & $\in[60,83.33]$ & - \\
\hline & $T_{S}$ & - & - & - & - & - \\
\hline
\end{tabular}

Numbers in cells are the theoretical predictions for the average prices $P$ in the corresponding market situation. States are $s_{1}: 3$ low-cost bidders, $s_{2}: 2$ low-cost bidders, $s_{3}: 1$ low-cost bidder, $s_{4}: 3$ high-cost bidders. Information treatments are $T_{B}$ : full info, $T_{D}$ : demand uncertainty, $T_{S}$ : supply uncertainty.

\footnotetext{
${ }^{35}$ Using $P_{\max }=50+\Delta=55$ as the first price which is profitable for a potential high-cost bidder.

${ }^{36}$ Using $P_{\max }=50+\Delta=55$.
} 


\section{Empirical results}

\subsection{Experimental design and procedure}

We implemented a uniform-price auction on a zTree platform (Fischbacher, 2007). We conducted three treatment variations, with the three information structures (full information, supply uncertainty and demand uncertainty) as the only treatment variable. In each session, the subjects were randomly and anonymously matched into groups of $n=3$ bidders forming a market. The group and the information structure remained unchanged during a session. A session lasted for about 24 periods. In all treatments, the computer always executed the following two random assignments at the beginning of each period. First, a level of market demand $\bar{Q} \in\{60,100,180\}$ was determined in each market with uniform probability $1 / 3$. Second, the computer randomly assigned one of the two possible technologies (1) to each bidder with probability $1 / 2$. Table 3 summarizes the resulting likelihoods of all possible market situations. Each bidder always knew the own technology in every period prior to bidding and independent of the information treatment. The three information

Table 3: Market situations: Probabilities of occurrence

\begin{tabular}{c|c|c|c|c}
\hline & $s_{1}$ & $s_{2}$ & $s_{3}$ & $s_{4}$ \\
& $3 \times c_{L}$ & $2 \times c_{L}, 1 \times c_{H}$ & $1 \times c_{L}, 2 \times c_{H}$ & $3 \times c_{H}$ \\
\hline $\bar{Q}=60$ & $1 / 24$ & $1 / 8$ & $1 / 8$ & $1 / 24$ \\
$\bar{Q}=100$ & $1 / 24$ & $1 / 8$ & $1 / 8$ & $1 / 24$ \\
$\bar{Q}=180$ & $1 / 24$ & $1 / 8$ & $1 / 8$ & $1 / 24$ \\
\hline
\end{tabular}

See (1) for the definition of $c_{L}, c_{H}$. Number in cells are the probabilities of occurrence.

structures were as follows.

- In the full information treatment, all technologies in a market and demand were common knowledge in each period prior to bidding.

- In the supply uncertainty treatment, only demand and the own technology were common knowledge in a market, while subjects had only the statistical information about technologies.

- In the demand uncertainty treatment, all technologies were common knowledge in a market, while subjects had only the statistical information about demand.

In each period, each subject first received the period and treatment-specific information about demand and technology. Then, every subject entered the number of units s/he would like to offer for each price ranging from 0 to 100 in steps of $\Delta=5$ in the current period. ${ }^{37}$ The computer only

\footnotetext{
${ }^{37}$ Subjects could enter their desired quantity at each price either by using a slider or by directly typing the number. Quantities had to be natural numbers no larger than the capacity of the subject's current capacity.
} 
accepted weakly increasing quantity numbers. After all three subjects in a market submitted their bids, the computer determined the uniform auction price and the equilibrium allocation in that period, using the pro-rata rationing protocol to split the residual quantity. ${ }^{38}$ Intuitively, pro-rata allocates the own offered quantity just below the auction price to each bidder plus possibly some residual quantity that depends on the relative bids at the auction price. At the end of each period, the market price, the own quantity sold, the own period profit and the supply schedules including costs of all subjects in the market were displayed to each subject. ${ }^{39}$

Experimental instructions were distributed in written form prior to the experiment. Additionally, a video tutorial was displayed. Subjects then could spend 10 minutes on a trial phase, where they could choose the supply schedules of all three bidders. This allowed them to test different strategies under different demand and cost constellations, and to familiarize themselves with the interface. Subsequently, subjects had to answer a test, assuring that all subjects understood the rules of the auction and the incentives (maximize own payoffs). No communication with other subjects was allowed, and the anonymity of participants' ID's was assured throughout each session. Sessions were conducted with university students between July 2015 and December 2016 at UNSW Australia, the Zurich University of Applied Sciences and the University of Zurich. Each session consisted of about 30 subjects, and each student participated in only one session and treatment. ${ }^{40}$ At the end of a session, one of the periods was randomly selected for payment, which consisted of the individual payoffs (points) realized in the respective period (converted into the local currency) and a lump-sum participation fee. ${ }^{41}$ On average, a session lasted about 2 hours, and participants earned around AUD 26 in Australia and CHF 45 in Switzerland.

\subsection{Main empirical results}

Table 4 shows the average prices of all treatment variations and all market situations $(s, \bar{Q})$. These averages, and the subsequent regression results, are obtained from stratified data, which limits the

\footnotetext{
${ }^{38}$ This is a standard protocol in empirical multi-unit auctions. See Brandts et al. (2014) and Appendix B.2 for the details. In practice, the National Electricity Market (NEM) in Australia invokes a very similar protocol.

${ }^{39}$ The idea for showing the bidding of all subjects was that this should facilitate the understanding of the auction, and strengthen the strategic thinking of the subjects. Such a procedure is not uncommon in experiments. E.g., Boone et al. (2012) also let all bidders in a Bertrand market (which is similar to a single-unit first-price auction) observe the competitors' costs and price bids by the end of each period. It is understood that if displaying such information has an effect, it is likely to foster competition (Huck et al., 2000). Note that because all probabilities were iid between periods, the issue of strategic learning with uncertainty, which matters in other auction environments (Bergemann and Horner, 2010), does not apply here. Moreover, learning seems no to be important; controlling for time periods does not affect any of our regression results in a significant way.

${ }^{40}$ Recruitment was conducted with ORSEE (Greiner, 2004). The subject pool consisted of Bachelor and Master students of quantitative subjects (including Economics and Business Administration).

${ }^{41}$ The conversion rates were AUD 6.70 per 1000 points in Australia and CHF 6.70 per 1000 points in Switzerland. The lump-sum participation fee (which insured subjects against losses) amounted to 4500 points in Australia and 6000 points in Switzerland.
} 
influence of (rare) outliers. ${ }^{42}$ Table 4 reveals sometimes substantial differences between the average prices across market situations and information treatments. We use a standard OLS framework

Table 4: Average price, by demand, competition state and treatment

\begin{tabular}{c|c|cc|cc|cc|cr}
\hline \hline & & \multicolumn{2}{|c|}{$s_{1}$} & \multicolumn{2}{c|}{$s_{2}$} & \multicolumn{2}{|c|}{$s_{3}$} & \multicolumn{2}{c}{$s_{4}$} \\
\hline \multirow{4}{*}{$\bar{Q}=60$} & $T_{B}$ & 15.96 & {$[8.13]$} & 23.06 & {$[29.74]$} & 47.03 & {$[14.04]$} & 59.26 & {$[15.70]$} \\
& $T_{D}$ & 17.37 & {$[13.03]$} & 24.5 & {$[28.84]$} & 43.21 & {$[31.09]$} & 61.05 & {$[15.43]$} \\
& $T_{S}$ & 15.4 & {$[7.04]$} & 22.24 & {$[19.24]$} & 36.34 & {$[29.22]$} & 56.25 & {$[7.77]$} \\
\hline \multirow{4}{*}{$\bar{Q}=100$} & $T_{B}$ & 18.96 & {$[10.83]$} & 31.23 & {$[32.33]$} & 56.98 & {$[28.01]$} & $95^{M}$ & {$[45.08]$} \\
& $T_{D}$ & 24.74 & {$[24.06]$} & 33.73 & {$[33.92]$} & 56.19 & {$[18.23]$} & 79.25 & {$[30.04]$} \\
& $T_{S}$ & 20.63 & {$[14.52]$} & 32.36 & {$[29.53]$} & 51.74 & {$[19.30]$} & 73.54 & {$[27.32]$} \\
\hline \multirow{4}{*}{$\bar{Q}=180$} & $T_{B}$ & 34.82 & {$[28.23]$} & 60.65 & {$[44.75]$} & 83.33 & {$[46.05]$} & 100 & \\
& $T_{D}$ & 32.63 & {$[22.24]$} & 59.17 & {$[36.65]$} & 83.5 & {$[45.56]$} & 100 & \\
& $T_{S}$ & 41.25 & {$[24.31]$} & 60 & {$[24.26]$} & 80.59 & {$[39.24]$} & 100 & \\
\hline \hline
\end{tabular}

Average prices with standard deviations in square brackets.

States are $s_{1}: 3$ low-cost bidders, $s_{2}: 2$ low-cost bidders, $s_{3}: 1$ low-cost bidder, $s_{4}: 3$ high-cost bidders. Information treatments are $T_{B}$ : full info, $T_{D}$ : demand uncertainty, $T_{S}$ : supply uncertainty.

$P=100$ in bottom right corner is an artifact of the experimental design. $M$ indicates "median price".

to assess these differences from the viewpoint of our empirical hypothesis. ${ }^{43}$ In all regressions, we adjust standard errors for clustering at the market level.

\subsubsection{Market power and information effects}

In this section, we test whether the data supports our central hypothesis that limited information should tend to lower average auction prices compared to full information.

Market power with full information We first assess hypothesis H1 regarding pivotal market power under full information. The middle part of columns " $s_{3}$ " and " $s_{4}$ " of Table 5 display the relevant regression outcomes for situations $\left(s_{3}, 100\right)$ and $\left(s_{4}, 100\right)$, and the bottom part of that table reports the respective statistical test results. The following illustration for situation $\left(s_{4}, 100\right)$ may help to interpret the table. The middle part of column " $s_{4}$ " implies that the average estimated price in situation $\left(s_{4}, 100\right)$ under full information is $59.259+24.312=83.571$. By the bottom part of column " $s_{4}$ ", the probability that the average price verifies $P \geq 80$ in situation $\left(s_{4}, 100\right)$ in the full information treatment (denoted as $P\left(s_{4}, 100 ; T_{B}\right) \geq 80$ in the table) is $p=0.824$. Such a large $p$-value indeed suggests that we cannot reject $P \geq 80$, consistent with H1. A more detailed analysis reveals that almost $50 \%$ of the prices even equal the maximal price of 100 . Indeed, the median price in $\left(s_{4}, 100\right)$ is $P_{m e d}=95$ (see Table 4 ), and a median regression (not reported) shows that

\footnotetext{
${ }^{42}$ The stratification aimed at excluding rare outlying prices that most likely are due to mistakes, such as $P=10$ in the full information monopoly setting $\left(s_{3}, 60\right)$. In sum, the stratification retains about $93 \%$ of the observations; see Table 12 in Appendix B for details. Using unstratified data does not change the main results qualitatively, but typically leads to noisier estimates with larger standard errors.

${ }^{43}$ Controlling for time (not reported) does not affect the empirical results. Further, we also use quantile regression, at the very least for robustness concerns.
} 
we cannot reject the null that $P_{\text {med }}=100(p$-value $=0.8) \cdot{ }^{44}$

Table 5: Supply uncertainty effects and market power $(\bar{Q} \in\{60,100\})$

\begin{tabular}{|c|c|c|c|c|}
\hline & $\left(s_{1}-s_{3}\right)$ & $\left(s_{1}-s_{4}\right)$ & $s_{3}$ & $s_{4}$ \\
\hline \multirow[t]{2}{*}{$T_{S}$} & $-3.408^{*}$ & $-3.895^{* *}$ & $-8.071 * * *$ & $-6.741^{* *}$ \\
\hline & $(1.761)$ & $(1.788)$ & $(1.835)$ & $(3.088)$ \\
\hline \multirow[t]{2}{*}{ Constant } & $36.448^{* * *}$ & $41.045^{* * *}$ & $52.226^{* * *}$ & $71.636^{* * *}$ \\
\hline & $(1.297)$ & $(1.361)$ & $(1.145)$ & $(2.503)$ \\
\hline \multirow[t]{2}{*}{$T_{S}$} & $-4.782^{* * *}$ & $-4.530^{* * *}$ & $-10.684^{* * *}$ & -3.009 \\
\hline & $(1.773)$ & $(1.656)$ & $(2.248)$ & $(1.959)$ \\
\hline \multirow[t]{2}{*}{$\bar{Q}_{100}$} & $8.971^{* * *}$ & $10.990 * * *$ & $9.948^{* * *}$ & $24.312^{* * *}$ \\
\hline & $(1.166)$ & $(1.186)$ & $(1.818)$ & $(3.288)$ \\
\hline \multirow[t]{2}{*}{$T_{S} \times \bar{Q}_{100}$} & $2.679^{*}$ & 1.256 & $5.448^{* *}$ & -7.021 \\
\hline & $(1.572)$ & $(1.603)$ & $(2.697)$ & $(4.561)$ \\
\hline \multirow[t]{2}{*}{ Constant } & $31.889^{* * *}$ & $35.459^{* * *}$ & $47.027 * * *$ & $59.259 * * *$ \\
\hline & $(1.253)$ & $(1.181)$ & $(0.936)$ & $(1.736)$ \\
\hline $\mathrm{N}$ & 690 & 793 & 291 & 103 \\
\hline Null & Tests H2a & & Tests H1,H2b & $\overline{\text { Tests } \mathrm{H} 1, \mathrm{H} 2 \mathrm{k}}$ \\
\hline$P\left(s_{3}, 100 ; T_{B}\right)=55$ & & & $p=0.27$ & \\
\hline$P\left(s_{3}, 100 ; T_{B}\right) \geq 60$ & & & $p=0.048$ & \\
\hline$P\left(s_{4}, 100 ; T_{B}\right) \geq 80$ & & & & $p=0.824$ \\
\hline$P\left(T_{S}\right) \geq P\left(T_{B}\right)$ & $p=0.029$ & $p=0.017$ & $p<0.01$ & $p=0.017$ \\
\hline$P\left(T_{S}, 60\right) \geq P\left(T_{B}, 60\right)$ & $p<0.01$ & $p<0.01$ & $p<0.01$ & $p=0.065$ \\
\hline$P\left(T_{S}, 100\right) \geq P\left(T_{B}, 100\right)$ & $p=0.152$ & $p=0.069$ & $p=0.011$ & $p=0.024$ \\
\hline
\end{tabular}

OLS regressions with clustered SE (market level). Unit of observation: Matching group. Dependent variable: Auction price. Independent variables: $T_{B}, T_{S}$ : treatment dummies indicating full information and supply uncertainty, respectively. First column: Pooled regression across states $s_{1}-s_{3}$. Second column: pooled regression across states $s_{1}-s_{4}$. Significance levels (two-sided tests): ${ }^{*} \mathrm{p}<0.1,{ }^{* *} \mathrm{p}<0.05,{ }^{* * *} \mathrm{p}<0.01$.

In case of situation $\left(s_{3}, 100\right)$, the middle part of column " $s_{3}$ " predicts an average price of $P=56.975$. The first two tests in the bottom part of column " $s_{3}$ " then show that i) we cannot reject $P=55(p$-value $=0.27)$, while ii $) P \geq 60$ is unlikely ( $p$-value $=0.048)$. Both findings are consistent with $\mathrm{H} 1$.

To test whether $P=100$ in situation $\left(s_{3}, 180\right)$, we use a median regression (not reported) to account for bunching at the price ceiling $P_{\max }=100$. The estimate price is $P_{\text {med }}=95$, with 95\%-CI $[91.1,98.9]$, which means that the price is close to but statistically still a bit below the theoretical prediction. Upon closer inspection, we find the reason to be that $P=55$ for about $20 \%$ of the observed prices, which cannot be explained by the SFE in Proposition 2, while $P=100$ in about $44 \%$ of all cases. If the median regression is trimmed to $P>55, P_{\text {med }}=100$ results. ${ }^{45}$ We conclude that a substantial portion of the data favors H1 in situation $\left(s_{3}, 180\right)$, acknowledging that the evidence for $\mathrm{H} 1$ is slightly weaker in $\left(s_{3}, 180\right)$ compared to the other market situations.

Result 1 The average estimated prices in market situations featuring full information and pivotal

\footnotetext{
${ }^{44}$ We apply the approach by Parente and Silva (2016) to calculate clustered robust standard errors also for the median regression.

${ }^{45}$ A Tobit regression (not reported) with upper limit 100 yields an estimated price of 93.1, and the hypothesis $P\left(s_{3}, 180\right)=100$ yields a $p$-value of 0.23 .
} 
power are mostly as conjectured by Hypothesis H1.

Hefti and Shen (2019) analyze the case of purely exclusive market power, as it occurs in situation $\left(s_{3}, 60\right)$ in detail. The single low-cost bidders fully exploits her market power, and captures the entire market at a price close to the marginal costs of the next-best competitor. ${ }^{46}$ In sum, the above empirical observations show that bidders strongly exert their structural market power in the full information treatment.

Supply uncertainty We now turn to hypothesis H2, according to which we should observe lower average prices with supply uncertainty. Figure 4 (LEFT) compares the distribution of auction prices for full information (gray) and supply uncertainty (red) for all states with at least one lowcost bidder $\left(s_{1}-s_{3}\right)$ and $\bar{Q} \in\{60,100\}$. The figure suggests a shift from high to low prices in case of supply uncertainty. This visual impression is corroborated by a pooled regression for states
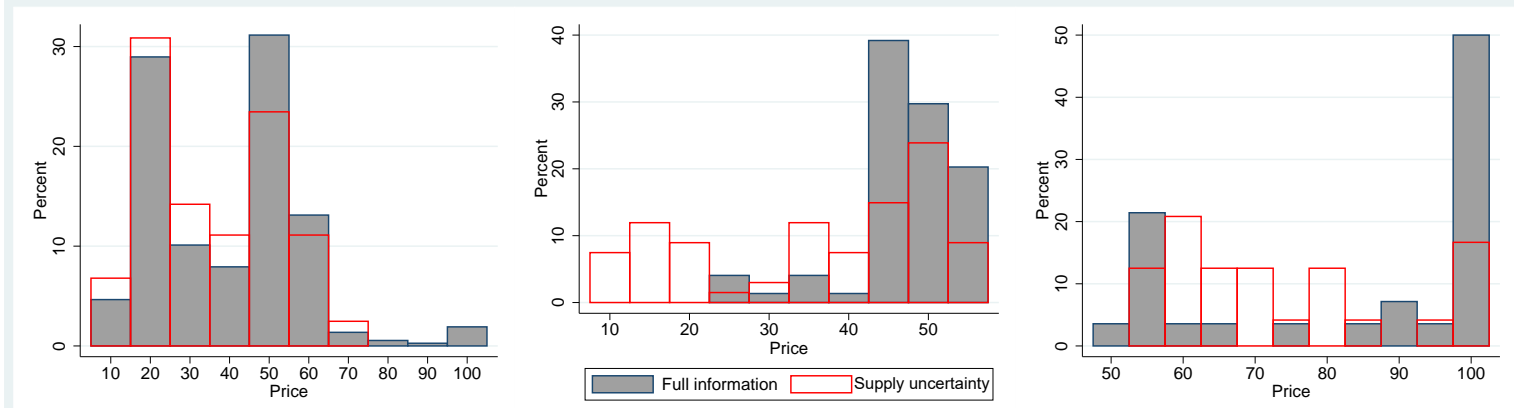

Figure 4: Supply uncertainty effects

LEFT: States $s_{1}-s_{3}$ and $\bar{Q} \in\{60,100\}$. MIDDLE: Exclusive market power $\left(s_{3}, 60\right)$. RIGHT: Pivotal market power $\left(s_{4}, 100\right)$.)

$s_{1}-s_{3}$ and $\bar{Q} \in\{60,100\}$, reported in the top of column 1, Table 5. The average price with supply uncertainty is 3.4 units lower than the respective average price with full information $(P=36.4)$. This difference is significant at the $5 \%$ level $(p$-value $=0.029$; bottom of Table 5$)$, and corresponds to a reduction in the markup $P-c$ by about $13 \%$ on average.

We check the robustness of this result in two ways. First, estimating the specification in the top of column 1 with quantile regression (not reported) yields a median prices of $P_{m e d}=40$ under full information, and of $P_{m e d}=30$ under supply uncertainty; the difference of 10 is significant at the $1 \%$ level. Second, we consider a regression that pools all states $s_{1}-s_{4}$ (column 2 ). This regression also estimates a lower average price in case of supply uncertainty ( $p$-value $=0.017$ ).

The middle part of Table 5 additionally distinguishes between $\bar{Q}_{60}$ and $\bar{Q}_{100}$. This analysis shows that the strictly lower average price under supply uncertainty is mainly caused by $\bar{Q}=60$.

\footnotetext{
${ }^{46}$ The average price is 47.027. Hefti and Shen (2019) show that the median price in this situation equals $P_{m e d}=45$, corresponding to a SFE, where the low-cost bidder wants to "play it safe".
} 
While the price is also lower under supply uncertainty for $\bar{Q}=100$, the difference of 2.1 units is not statistically significant $(p$-value $=0.152)$. In any case, we can soundly reject that supply uncertainty induces higher auction prices, consistent with Hypothesis H2a.

We now turn to Hypothesis H2b. The distribution of prices is illustrated in Figure 4 in situations $\left(s_{3}, 60\right)$ (MIDDLE) and $\left(s_{4}, 100\right)($ RIGHT). In both cases, the price distribution is clearly shifted towards lower prices in case of supply uncertainty. The corresponding statistical evidence is presented in columns 3 and 4 of Table 5 . In situations $\left(s_{3}, 60\right)$ and $\left(s_{3}, 100\right)$, supply uncertainty leads to significantly lower prices, and thus breaks the exclusive market power exerted by the low-cost bidder under full information. Likewise, supply uncertainty breaks the symmetric pivotal market power of the high-cost bidders in $\left(s_{4}, 100\right)$ under full information. The latter becomes even more evident, if we use a median regression (not reported) instead of OLS. ${ }^{47}$ We find $P_{\text {med }}=70$ with supply uncertainty, which is 25 units below the full information median price $\left(P_{\text {med }}=95\right)$; the difference is significant at the $1 \%$ level. Thus, we conclude that supply uncertainty breaks both pivotal and exclusive market power as predicted by H2b. Additionally, we find evidence for lower average prices with supply uncertainty even in the situations with only high-cost bidders (last column of Table 5).

Result 2 Supply uncertainty leads to significantly lower average prices, consistent with Hypothesis H2 in the strict sense. Specifically, the markup is reduced by about $13 \%$ averaged over all situations involving at least one low-cost bidder and $\bar{Q} \in\{60,100\}$. Further, supply uncertainty breaks both exclusive and pivotal market power, with a reduction of about $29 \%$ in average markup in case of purely exclusive market power $\left(s_{3}, 60\right)$, and a reduction of about $56 \%$ in the median markup in case of purely pivotal market power $\left(s_{4}, 100\right)$.

Demand uncertainty We proceed similarly to test the effects of demand uncertainty (Hypothesis H3). The OLS results are summarized in Table 6. In the bottom part of column 1, Table 6 , we test whether demand uncertainty leads to (weakly) lower average prices in a pooled OLS regression over all demand levels and both states $s_{2}, s_{4}$ as claimed by H3a. The null that the price is higher with demand uncertainty is rejected ( $p$-value 0.49$)$. This $p$-value also indicates that the price is also not strictly lower under demand uncertainty, in contrast to our observation with supply uncertainty. A similar pattern is revealed by column 2 , where $s_{2}$ and $s_{4}$ are discerned. The data thus suggests that the average price under demand uncertainty and full information are statistically indistinguishable, thereby confirming hypothesis H3a in its weak sense.

For H3b, a median regression (not reported) is more appropriate than OLS in case of $\left(s_{4}, 100\right)$,

\footnotetext{
${ }^{47}$ This makes particularly sense in view of the observed bunching at $P=100$ under full information.
} 
Table 6: Demand uncertainty effects

\begin{tabular}{cccc}
\hline \hline & $\left(s_{2}, s_{4}\right)$ pooled & $s_{2}, s_{4}$ & $s_{3}$ \\
\hline$T_{D}$ & 0.048 & 0.509 & -1.759 \\
& $(2.371)$ & $(2.499)$ & $(2.018)$ \\
$s_{4}$ & & $32.983^{* * *}$ & \\
$T_{D} \times s_{4}$ & & $(1.910)$ & \\
& & -1.761 & \\
Constant & & $(3.241)$ & \\
& & $38.653^{* * *}$ & $63.159^{* * *}$ \\
$\mathrm{~N}$ & $(1.808)$ & $(1.782)$ & $(1.386)$ \\
$\mathbf{N u l l}$ & 518 & 518 & 414 \\
\hline$P\left(T_{B}\right) \geq P\left(T_{D}\right)$ & Tests H3a & Tests H3a & Tests H3b \\
$P\left(T_{B} ; s_{2}\right) \geq P\left(T_{D} ; s_{2}\right)$ & 0.49 & & \\
$P\left(T_{B} ; s_{4}\right) \geq P\left(T_{D} ; s_{4}\right)$ & & 0.42 & 0.388 \\
$P\left(T_{D}\right)=P\left(T_{B}\right)$ & & & 0.64 \\
\hline \hline
\end{tabular}

OLS regressions with clustered SE (market level). Unit of observation: Matching group. Dependent variable: Auction price. Independent variables: $T_{B}, T_{D}$ : treatment dummies indicating full information and demand uncertainty, respectively. First column: Pooled regression across all demand levels and states $s_{2}, s_{4}$. Second column: Pooled regression across all demand levels. Third column: pooled regression across all demand levels for $s_{3}$. Significance levels (two-sided tests): ${ }^{*} \mathrm{p}<0.1,{ }^{*} \mathrm{p}<0.05,{ }^{* * *} \mathrm{p}<0.01$.

as full information prices are bunched at $P=100$. Consistent with $\mathrm{H} 3 \mathrm{~b}$, we find that $P_{\text {med }}=80$ in case of demand uncertainty, which is strictly below its full information counterpart of $P_{\text {med }}=95$ $(p$-value $=0.09)$. Finally, column 3 of Table 6 shows that we cannot reject the equality of average prices in the monopoly state $s_{3}$. We find the same result in OLS and median regressions if all three demand levels are discerned (not reported), supporting H3b.

Result 3 Average prices in states $s_{2}, s_{4}$ with demand uncertainty are statistically indistinguishable from the corresponding full information prices, which matches Hypothesis H3a in the weak sense. Consistent with H3b, prices in the monopoly state $s_{3}$ are similar with demand uncertainty and full information, and the median price in situation $\left(s_{4}, 100\right)$ is strictly lower than its full information counterpart.

Allocative efficiency We also consider whether limited information affects the allocative efficiency of the auction. Such inefficiency would occur if low-cost bidders share the market with high-cost bidders when low-cost bidders themselves are able to seize the entire market. The fact that, by $\mathrm{H} 2$ and H3, limited information should make biding more competitive, suggests that the allocative efficiency does not diminish under limited information. Indeed, we find that the median supply of high-cost bidders in situations $\left(s_{2}, 60\right),\left(s_{3}, 60\right)$ and $\left(s_{2}, 100\right)$ is zero in all information treatments. In other words, the low-cost bidders seize the market in all information treatments. 


\subsubsection{Demand and competition effects}

Table 4 suggests that more demand or a decrease in competition leads to higher average prices in each information treatment, as conjectured in Hypothesis H4. We test H4 formally by running regressions for each information treatment, using an OLS dummy variable specification to distinguish between the competition states and the demand levels, with situation $\left(s_{1}, 60\right)$ as base group. The outcome is reported in the three columns of Table 7 . The following illustrative calculations

Table 7: Competition effects by information structure

\begin{tabular}{|c|c|c|c|}
\hline & Full & Supply & Demand \\
\hline \multirow[t]{2}{*}{$s_{2}$} & $9.40^{* * *}$ & $6.84^{* * *}$ & $6^{* *}$ \\
\hline & $(2.44)$ & $(1.32)$ & $(2.04)$ \\
\hline \multirow[t]{2}{*}{$s_{3}$} & $31.82^{* * *}$ & $20.94^{* * *}$ & $29.54^{* * *}$ \\
\hline & $(1.51)$ & $(2.03)$ & $(3.14)$ \\
\hline \multirow[t]{2}{*}{$s_{4}$} & $44.95^{* * *}$ & $40.85^{* * *}$ & $44^{* * *}$ \\
\hline & $(2.06)$ & $(1.01)$ & $(2.23)$ \\
\hline \multirow[t]{2}{*}{$\bar{Q}_{100}$} & $3.45^{* *}$ & $5.23^{* * *}$ & $8^{*}$ \\
\hline & $(1.61)$ & $(1.51)$ & $(3.98)$ \\
\hline \multirow[t]{2}{*}{$\bar{Q}_{180}$} & $19.91^{* * *}$ & $25.85^{* * *}$ & $14.5^{* * *}$ \\
\hline & $(3.76)$ & $(3.13)$ & $(3.71)$ \\
\hline \multirow[t]{2}{*}{$s_{2} \times \bar{Q}_{100}$} & $4.69^{*}$ & $4.90^{*}$ & 4.5 \\
\hline & $(2.69)$ & $(2.49)$ & $(4.52)$ \\
\hline \multirow[t]{2}{*}{$s_{2} \times \bar{Q}_{180}$} & $16.65^{* *}$ & $11.91^{* * *}$ & $24.83^{* * *}$ \\
\hline & $(5.92)$ & $(3.39)$ & $(5.87)$ \\
\hline \multirow{2}{*}{$s_{3} \times \bar{Q}_{100}$} & $6.53^{* * *}$ & $10.17^{* * *}$ & 2.13 \\
\hline & $(2.14)$ & $(2.37)$ & $(4.59)$ \\
\hline \multirow[t]{2}{*}{$s_{3} \times \bar{Q}_{180}$} & $16.08^{* * *}$ & $18.40^{* * *}$ & $20.96^{* * *}$ \\
\hline & $(3.42)$ & $(3.84)$ & $(6.26)$ \\
\hline \multirow[t]{2}{*}{$s_{4} \times \bar{Q}_{100}$} & $22.56^{* * *}$ & $12.07^{* * *}$ & 9 \\
\hline & $(3.73)$ & $(3.61)$ & $(6.83)$ \\
\hline \multirow[t]{2}{*}{ Constant } & $15.88^{* * *}$ & $15.4^{* * *}$ & $16^{* * *}$ \\
\hline & $(1.23)$ & $(0.81)$ & $(2.89)$ \\
\hline$N$ & 413 & 540 & 228 \\
\hline Null & \multicolumn{2}{|c|}{ Competition effect } & \\
\hline$P\left(s_{1}, 100\right) \geq P\left(s_{2}, 100\right)$ & $p<0.01$ & $p<0.01$ & \\
\hline$P\left(s_{2}, 60\right) \geq P\left(s_{3}, 60\right)$ & & $p<0.01$ & \\
\hline$P\left(s_{2}, 100\right) \geq P\left(s_{3}, 100\right)$ & & $p<0.01$ & \\
\hline Null & Dema & ect & \\
\hline$P\left(s_{2}, 60\right) \geq P\left(s_{2}, 100\right)$ & $p<0.01$ & $p<0.01$ & $p<0.01$ \\
\hline$P\left(s_{4}, 60\right) \geq P\left(s_{4}, 100\right)$ & $p<0.01$ & & $p<0.01$ \\
\hline$P\left(s_{1}, 100\right) \geq P\left(s_{1}, 180\right)$ & $p<0.01$ & & \\
\hline$P\left(s_{3}, 60\right) \geq P\left(s_{3}, 100\right)$ & & $p<0.01$ & \\
\hline$P\left(s_{2}, 100\right) \geq P\left(s_{2}, 180\right)$ & & & $p<0.01$ \\
\hline
\end{tabular}

OLS regressions with clustered SE (market level). Unit of observation: Matching group.

Dependent variable: Auction price. Independent variables: " $s_{2}, s_{3}, s_{4}$ ": state dummies; " $\bar{Q}_{100}, \bar{Q}_{180}$ ": demand dummies; Constant: $\left(s_{1}, 60\right) . s_{4} \times \bar{Q}_{180}$ was omitted because $P=100$ by design in this situation. Significance levels (two-sided tests): ${ }^{*} \mathrm{p}<0.1,{ }^{* *} \mathrm{p}<0.05,{ }^{* * *} \mathrm{p}<0.01$.

should facilitate its reading. The first column presents results for the full information treatment (Hypothesis H4a). We can directly assess whether $P\left(s_{1}, 60\right)<P\left(s_{2}, 60\right)$ from the first entry in the column, which shows that the price increases significantly (1\% level) by 9.4 if an additional 
low-cost competitor is present and $\bar{Q}=60 .{ }^{48}$ Similarly, the average price increases significantly (5\% level) by 3.45 if demand increases from 60 to 100 in presence of a total of three low-cost competitors (state $s_{1}$ ). We can test the remaining claims in hypothesis H4a by appropriately combining the regression results; the respective test outcomes are in the bottom part of the column. To test whether $P\left(s_{1}, 100\right)<P\left(s_{2}, 100\right)$, we seek to reject the null that $P\left(s_{1}, 100\right) \geq P\left(s_{2}, 100\right)$ with sufficient confidence. The corresponding $p$-value is $<0.01$, meaning that the null can be rejected almost certainly. ${ }^{49}$ Likewise, to test whether $P\left(s_{2}, 60\right)<P\left(s_{2}, 100\right)$, we seek to reject the null that $P\left(s_{2}, 60\right) \geq P\left(s_{2}, 100\right)$. The corresponding $p$-value of $<0.01$ again allows us to reject the null in favor of $P\left(s_{2}, 60\right)<P\left(s_{2}, 100\right)$. Hypotheses $\mathrm{H} 4 \mathrm{~b}$ and $\mathrm{H} 4 \mathrm{c}$ are tested in columns 2 and 3 , respectively, of Table 7 . We conclude from this analysis that $\mathrm{H} 4$ is entirely supported by the data.

Result 4 Average prices increase in demand and decrease in the number of effective competitors as predicted by Hypothesis H4. Averaged across all information treatments and demand levels, adding an additional competitor $\left(s_{2} \rightarrow s_{1}\right)$ reduces the markups earned by low-cost competitors by 50\%. Averaged across all information treatments and states $s_{1}, s_{2}$, a $10 \%$ increase in demand increases markups of low-cost bidders by $15.9 \%$.

\subsubsection{Supra-competitive equilibria}

Regarding Hypothesis H5, Table 4 directly reveals that all average prices are above their respective lower bounds given in Table 2. Hence the probabilities of observing prices at least as extreme as these lower bounds must be above 0.5. The data thus is consistent with the supra-competitive equilibrium prediction under full information, as well as with the increasing bounds as predicted by A-LinSFE in case of supply and demand uncertainty. This result is also confirmed if the means in Table 4 are replaced by medians (output omitted). We next assess with pooled regressions whether the average prices under supply and demand uncertainty reside in the intervals in the right column and bottom row of Table 2 , respectively. With supply uncertainty, the estimated average price for $\bar{Q}=60$ is 27.1 , with $95 \%$ CI [24.5,29.7], and for $\bar{Q}=100$ it is 38.6 with $95 \%$ CI $[36.2,41.0]$. Thus, the pooled average price matches well with the A-LinSFE prediction for $\bar{Q}=60$, but is too high in case of $\bar{Q}=100$. With demand uncertainty, the estimated average price for states $s_{2}, s_{4}$ is 36.6 with $95 \%$ CI $[32.7,40.4]$, and 70.3 with $95 \%$ CI $[65.6,75.1]$, respectively. The pooled average price thus fits well with the A-LinSFE prediction in state $s_{4}$, and is rather close to the upper bound for state $s_{2}$. The above evidence can be summarized as follows.

\footnotetext{
${ }^{48}$ Recall that Hypothesis H4 is of a one-sided nature. In the example, the null (which we seek to reject) thus is $P\left(s_{1}, 60\right) \geq P\left(s_{2}, 60\right)$, meaning that we can divide the corresponding $p$-values by 2 .

${ }^{49}$ In the regression specification of Table 7 , this null states that $s_{2}+s_{2} \times \bar{Q}_{100} \leq 0$, from which the $p$-value can be derived.
} 
Result 5 All average prices are above their respective lower bounds in Table 2, consistent with H5. In case of supply and demand uncertainty, the average prices reside within the predicted price intervals of Table 2, except for $\bar{Q}=100$ with supply uncertainty, where the price is too high.

\subsection{Bidding behavior}

In competitive situations, the SFE analysis reveals that the parameters $\alpha, \beta$ of all LinSFE or ALinSFE verify certain equilibrium constraints. While the theory predicts multiple equilibria, we can use restrictions (3), (12) and (22) to estimate the average empirical supply function $q(P)=\hat{\alpha}^{*}+\hat{\beta}^{*} P$ with a Constrained Least Squares (CLS) approach. ${ }^{50}$ Specifically, we use CLS to estimate the parameters $(\alpha, \beta)$ of the supply functions for the competitive situations in the full information treatment, as well as for the two uncertainty treatments. In the latter cases, we estimate on the bidding data pooled across all situations which bidders cannot distinguish due to their limited information. The CLS estimator selects among all linear supply functions verifying the respective equilibrium restriction the one that best describes the average bidding behavior.

We use the resulting estimates in three ways to assess whether (A)-LinSFE delivers a sensible description of the equilibrium bidding behavior on average. First, the estimated average bid function yields a predicted price $\hat{P}_{C L S}$, which must be statistically identical with the true average price $P^{*}$, provided that (A)-LinSFE is an adequate description of equilibrium bidding behavior. Additionally, we can compare $\hat{P}_{C L S}$ to the price $\hat{P}_{O L S}$ predicted from a standard OLS estimation of the same data. Intuitively, the restriction imposed by (A)-LinSFE increases efficiency of the estimator, provided that the restriction itself is empirically adequate. Thus, if (A)-LinSFE is a reasonable behavioral framework, then the CLS prices should not perform worse than their OLS counterparts in predicting the true average prices. Second, we can check whether the CLS estimates of $\beta^{*}$ are consistent with the respective bounds given by (6), (17) and (27). Third, we can verify whether the estimated $\beta^{*}$ match the requirements in Corollaries 2 and 3 that are needed to support the competition and demand effects on auction prices as observed in the data.

Figure 5 exemplarily shows the estimated supply functions using CLS and OLS for the three information treatments. The CLS estimates of the supply functions (black lines), together with their 95\% CI's reside within the theoretical bounds (blue lines). Moreover, the true average price lies within the 95\%-CI of the predicted CLS-price, while OLS seems less accurate in this respect. A direct comparison of the price estimates, as visualized by Figure 6 in case of full information, indicates that the CLS prices indeed are closer than the OLS prices to the true average prices.

The graphical impressions in Figures 5 and 6 are corroborated by statistical evidence. Table

\footnotetext{
${ }^{50}$ See, e.g., Greene (2012) for a general treatment of this estimator.
} 

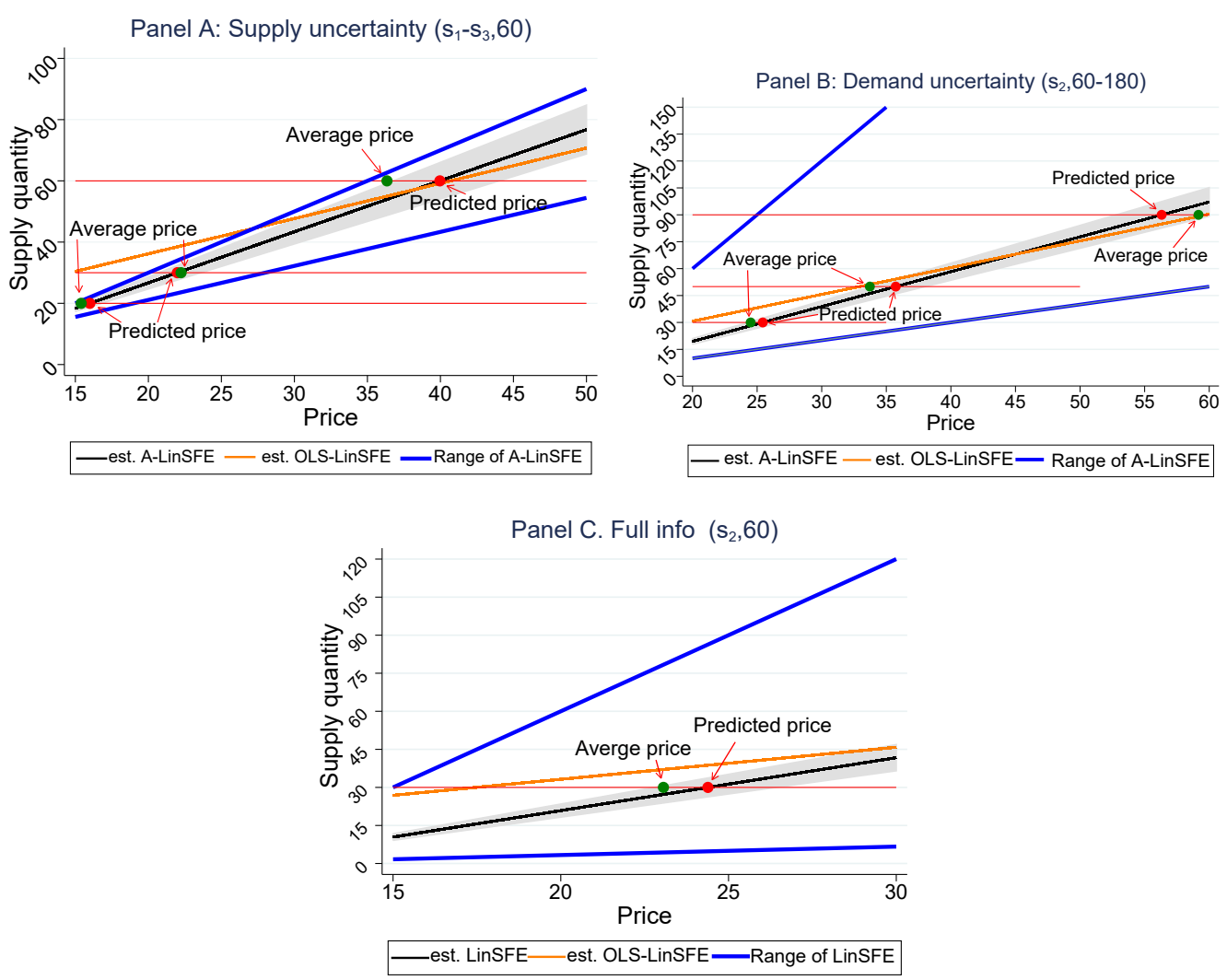

Figure 5: Constrained Linear SFE estimation

Figure 5 plots the CLS estimation of LinSFE in various cases with their 95\%-CI's (gray areas). Blue lines are the boundaries of all possible (A)-LinSFE. Red dots are market prices predicted by the estimated (A-)LinSFE for CLS, and green dots mark the observed average market prices. Orange lines correspond to the (unrestricted) OLS estimation on the same data.

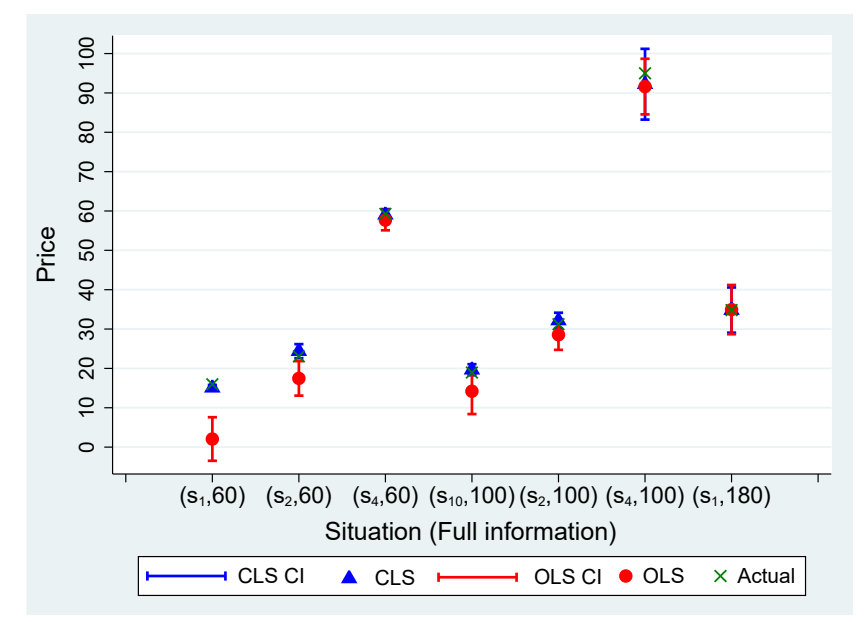

Blue triangles are CLS price predictions, red dots are (unrestricted) OLS price predictions, green crosses are true average prices. Blue and red intervals are the respective $95 \%$ confidence intervals.

Figure 6: CLS and OLS price predictions (full information)

8 presents the CLS estimation of the supply function $\alpha+\beta P$ for the full information treatment, jointly with the predicted prices obtained from CLS and OLS estimations using exactly the same 
data. The middle part of Table 8 directly shows that $\hat{P}_{C L S}$ is closer to $P^{*}$ than $\hat{P}_{O L S}$ in all cases.

Table 8: CLS Supply function estimation: Full information

\begin{tabular}{c|ccc|ccc|c}
\hline \hline & $\left(s_{1}, 60\right)$ & $\left(s_{2}, 60\right)$ & $\left(s_{4}, 60\right)$ & $\left(s_{1}, 100\right)$ & $\left(s_{2}, 100\right)$ & $\left(s_{4}, 100\right)$ & $\left(s_{1}, 180\right)$ \\
\hline$\hat{\alpha}^{*}$ & -9.70 & -20.85 & -45.26 & -0.59 & -22.55 & -3.07 & 17.91 \\
& $(1.43)$ & $(1.32)$ & $(1.73)$ & $(1.28)$ & $(1.03)$ & $(2.14)$ & $(1.43)$ \\
$\hat{\beta}^{*}$ & 1.97 & 2.09 & 1.11 & 1.73 & 2.26 & 0.39 & 1.21 \\
& $(0.14)$ & $(0.13)$ & $(0.09)$ & $(0.13)$ & $(0.1)$ & $(0.04)$ & $(0.14)$ \\
{$[\beta, \bar{\beta}]$} & {$\left[\frac{1}{4}, 2\right]$} & {$\left[\frac{3}{4}, 6\right]$} & {$\left[\frac{1}{5}, 2\right]$} & {$\left[\frac{5}{12}, \frac{10}{3}\right]$} & {$\left[\frac{5}{4}, 10\right]$} & {$\left[\frac{1}{3}, \frac{10}{3}\right]$} & {$\left[\frac{3}{4}, 6\right]$} \\
\hline $\mathrm{N}$ & 672 & 1344 & 924 & 756 & 1512 & 1008 & 756 \\
\hline$\hat{P}_{C L S}$ & 15.08 & 24.39 & 59.05 & 19.66 & 32.17 & 92.21 & 34.82 \\
& $(0.37)$ & $(0.91)$ & $(0.74)$ & $(0.72)$ & $(1.01)$ & $(4.59)$ & $(2.93)$ \\
$P^{*}$ & 15.96 & 23.06 & 59.26 & 18.96 & 31.23 & $95^{M}$ & 34.82 \\
$\hat{P}_{O L S}$ & 2.06 & 17.47 & 57.67 & 14.21 & 28.55 & 91.61 & 34.94 \\
& $(2.82)$ & $(2.24)$ & $(1.32)$ & $(2.97)$ & $(1.96)$ & $(3.61)$ & $(3.19)$ \\
\hline Tests & & & & & & & \\
$\hat{P}_{C L S}=P^{*}$ & 0.38 & 0.53 & 0.91 & 0.63 & 0.67 & 0.61 & 0.99 \\
$\hat{P}_{O L S}=P^{*}$ & $<0.01$ & 0.06 & 0.47 & 0.14 & 0.35 & 0.51 & 0.98 \\
\hline \hline
\end{tabular}

Unit of observation: Subject. $\hat{\alpha}^{*}$ and $\hat{\beta}^{*}$ are estimated parameters of LinSFE, and $\hat{P}_{C L S}$ is the predicted price by the CLS estimates, with clustered SE in parentheses. Restriction (3) was used state-wise. $[\beta, \bar{\beta}]$ is the range of admissible values for $\beta$ in LinSFE obtained from (6). $\hat{P}_{O L S}$ is the predicted price by the OLS estimates of $\alpha+\beta P$ for the same data. $P^{*}$ is the true average price. For $s<4(=4)$ only bids of low-cost (high-cost) types were estimated. The last two rows reported the p-values of t-tests for the null hypotheses of no significant difference between the predicted prices and the true average prices. ${ }^{M}$ Median used (instead of mean) due to data concentration at $P=100$.

Moreover, the large $p$-values in the bottom part of Table 8 imply that the predicted CLS prices are statistically indistinguishable from the true average prices, while this is not always true for OLS prices. The most dramatic deviation occurs in situation $\left(s_{1}, 60\right)$, where the OLS estimation yields a predicted price of $P=2.06$, which is very far from the actual mean price of $15.96 .^{51}$

The same conclusion holds for the two uncertainty treatments. With supply uncertainty, the relevant A-LinSFE estimates $\left(\hat{\alpha}^{*}, \hat{\beta}^{*}\right)$ are obtained by estimating $q=\alpha+\beta P$ from individual bidding data of all low-cost bidders pooled across states $s_{1}-s_{3}$, conditional on restriction (12). Then, the predicted average price $\hat{P}_{C L S}$ follows from using $\hat{\beta}^{*}$ in (14). Likewise, in case of demand uncertainty, $\left(\hat{\alpha}^{*}, \hat{\beta}^{*}\right)$ are obtained by estimating $q=\alpha+\beta P$ from individual bidding data of all low-cost bidders in state $s_{2}$, and all high-cost bidders in state $s_{4}$, pooled across demand levels, conditional on restriction (22). Then, $\hat{P}_{C L S}$ is obtained by using the respective $\hat{\beta}^{*}$ in $(24)$. The regression results are summarized in Appendix B.3, Tables 9 and 10. In both cases, $\hat{P}_{C L S}$ is strictly closer to the true average price $P^{*}$ (pooled across the market situations that cannot be discerned by bidders) than the corresponding $\hat{P}_{O L S}$.

There is another consistency test for A-LinSFE as an equilibrium concept: We can check whether the state-wise prices implied by the CLS estimation also match the true average prices in each state

\footnotetext{
${ }^{51}$ Specifically, OLS yields $q(P)=17.55+1.19 P$ which, by (3), cannot be part of a LinSFE.
} 
separately (rather than just averaged across all relevant states). Notably, this hypothesis could fail, despite a match of the CLS prediction of the average pooled price with the corresponding true average price, if the average bidding function displays sufficient non-linearity (contradicting A-LinSFE). As we show in Appendix B.3 (Tables 9 and 10), the data confirms that the state-wise CLS prices statistically range close to the average true state-wise prices, speaking in favor of the A-LinSFE concept.

Further, Table 8 shows that all estimates for $\hat{\beta}^{*}$ are consistent with the admissible values of $\beta$ in LinSFE as identified by (6). ${ }^{52}$ The same holds for the two uncertainty treatments (see Tables 9 - 10). Finally, we assess whether the estimates of $\beta^{*}$ match the comparative-static requirements of Corollaries 2 and 3 as $m$ or $\bar{Q}$ increases. The corresponding analysis is summarized in Table 11 of Appendix B.3, showing that this indeed is the case. Hence the average bidding behavior, as estimated by CLS, responds to changes in $m$ and $\bar{Q}$ in a way that is consistent with the sufficient conditions required to explain the observed patterns of the auction prices.

Result 6 With full information the predicted CLS prices $\hat{P}_{C L S}$ are closer to the true average price $P^{*}$ than $\hat{P}_{O L S}$. Likewise, averaged over all respective market situations, $\hat{P}_{C L S}$ is closer to $P^{*}$ than $\hat{P}_{O L S}$ in case of supply and demand uncertainty. Additionally, all state-wise prices predicted by $C L S$ are statistically close to the true average state-wise prices in both uncertainty treatments. The estimated values of $\beta$ are consistent with the bounds identified by the (A)-LinSFE approach in all information treatments. Finally, $\hat{\beta}^{*}$ as estimated by $C L S$, varies with $m$ and $\bar{Q}$ in a way consistent with the empirical patterns of the true average prices $P^{*}$.

\subsection{Practitioners vs. student subjects}

To assess the reliability of the bidding data generated by student subjects, we conducted a limited session with practitioners, some of which were former electricity traders. ${ }^{53}$ The main aim was to see whether the bidding behavior of practitioners deviates in a meaningful way from student behavior. We did not detect such a statistical difference, see Figure 7 (Appendix B.5) for an illustration.

\section{Conclusion}

This article studied market power and competition in a supply-side uniform-price multi-unit auction under different information structures, featuring either idiosyncratic or aggregate market un-

\footnotetext{
${ }^{52}$ The intervals $[\underline{\beta}, \bar{\beta}]$ are obtained from (6) with parameter values $\bar{Q} \in\{60,100,180\}, m \in\{1,2\}$, and $c=10$, $\bar{P}=50$ for states $s_{1}$ and $s_{2}$, and $c=50, \bar{P}=100$ for state $s_{4}$.

${ }^{53}$ The session was conducted at Swissgrid, the Swiss electricity transition grid operator, with 14 traders in the full information and supply uncertainty treatment. We did not use monetary incentives, but instead Swissgrid awarded three physical prices to the best three traders (a bottle of champagne, Swiss chocolate and alike).
} 
certainty. Specifically, we considered the cases of complete information, private but perfect cost information in an independent values setting, and symmetric but incomplete information about market demand. To our knowledge, no previous study has attempted to elicit how these various information structures interact with the exerted market power and competition in the context of a multi-unit auction, and what the resulting consequences for market prices and allocative efficiency are. Our design encompasses both exclusive and pivotal market power, as well as different levels of competition as measured by the number of symmetric competitors on the relevant price domains. The experimental approach gives us the necessary degree of control to assure that any observed change in auction outcome can be attributed to the changes in the information structure.

We found that average prices are not higher under both forms of uncertainty. In case of supply uncertainty, the average prices were even significantly, and substantially, below their full information counterparts. This contrasts with the theoretical prediction of the single-unit auction case, according to which there should be no difference in auction prices and revenues on average. It is already known that multi-unit auctions differ in important respects from their single-unit counterparts. For example, Ausubel et al. (2014) show that revenue equivalence across different auction formats or truthful bidding do not extend to the multi-unit auction case as a consequence of strategic differential bid shading, which is intrinsic to multi-unit auctions. Likewise, the "linkage principle", according to which more information about competitors should lead to more competitive bidding in a single-unit first-price auction with affiliated values (Milgrom and Weber, 1982), does not extend to the multi-unit auction case (Perry and Reny, 1999). ${ }^{54}$ Our finding suggests to add the outcome equivalence between private and complete information to the list of properties that seem not to transfer easily from the single-unit to the multi-unit auction case.

More generally, our findings caution against market policies aimed blindly at increasing "market transparency" about the various competitors. If such policies mainly have the effect of increasing the knowledge of competitors about each other, this could result in a less competitive auction outcome according to our results. In this respect, the influence of idiosyncratic information on the multi-unit auction outcome is not fully explored by this article. In particular, we have not studied the case of correlated costs, nor have we considered that the knowledge about the own possibilities could be noisy. Theoretical research indicates that improvements in the idiosyncratic information about the own technology may have a pro-competitive equilibrium effect (Vives, 2011; Holmberg and Wolak, 2015). A further limitation of our study, as with most experiments, is that we needed to assume specific and parametrized cost functions. It would be interesting to see future

\footnotetext{
${ }^{54}$ As emphasized by Perry and Reny (1999) or Buchanan et al. (2016), the linkage principle has sometimes been (sloppily) equated with a general sentiment that increasing bidder-side information tends to increase auction revenues and efficiency by making strategic bidding more competitive.
} 
empirical studies that assess whether the pro-competitive effect of supply uncertainty we determine is robust to other multi-unit auction settings. Likewise, future studies could seek to disentangle the effects of better information about the own technology, as opposed to better information about the competitors.

Finally, the broad idea behind our behavioral equilibrium concept in case of the two uncertainty treatments (A-LinSFE), stating that bidders play as if they faced the average market situation with certainty, could be studied as an alternative to the cognitively demanding structure imposed by Bayesian Nash Equilibria in other strategic models, such as Cournot or Bertrand competition.

\section{References}

Andreoni, J., Y.-K. Che, And J. Kim (2007): “Asymmetric information about rivals' types in standard auctions: An experiment," Games and Economic Behavior, 59, 240-259.

Armantier, O. And E. SBai (2006): "Estimation and comparison of treasury auction formats when bidders are asymmetric," Journal of Applied Econometrics, 21, 745-779.

Ausubel, L. M., P. Cramton, M. Pycia, M. Rostek, And M. Weretka (2014): "Demand reduction and inefficiency in multi-unit auctions," The Review of Economic Studies.

Baldick, R., R. Grant, And E. Kahn (2004): "Theory and application of linear supply function equilibrium in electricity markets," Journal of regulatory economics, 25, 143-167.

Bergemann, D., B. Brooks, And S. Morris (2017): "First-Price Auctions With General Information Structures: Implications for Bidding and Revenue," Econometrica, 85, 107-143.

Bergemann, D. And J. Horner (2010): "Should auctions be transparent?" Working paper.

Blume, A. (2003): "Bertrand without fudge," Economics Letters, 78, 167-168.

Bolle, F., V. Grimm, A. Ockenfels, And X. Del Pozo (2013): "An experiment on supply function competition," European Economic Review, 63, 170-185.

Boone, J., M. J. L. Aylwin, W. Müller, And A. R. Chaudhuri (2012): "Bertrand competition with asymmetric costs: Experimental evidence," Economics Letters, 117, 134-137.

Borenstein, S., J. B. Bushnell, And F. A. Wolak (2002): "Measuring market inefficiencies in California's restructured wholesale electricity market," The American Economic Review, 92, 1376-1405.

Bosco, B., L. PARIsio, AND M. Pelagatti (2012): "Strategic bidding in vertically integrated power markets with an application to the Italian electricity auctions," Energy Economics, 34, 2046-2057.

Brandts, J., S. S. Reynolds, And A. Schram (2014): "Pivotal Suppliers and Market Power in Experimental Supply Function Competition," The Economic Journal, 124, 887-916.

Buchanan, J., S. Guerstad, and D. Porter (2016): "Information Effects in Uniform Price Multi-Unit Dutch Auctions," Southern Economic Journal, 126-145.

Cassola, N., A. Horta $\tilde{\S} \S s u$, And J. Kastl (2013): "The 2007 Subprime Market Crisis Through the Lens of European Central Bank Auctions for Short-Term Funds," Econometrica, 81, 1309-1345.

Davis, D. D. And C. A. Holt (2008): "The Exercise of Market Power in Laboratory Experiments," Handbook of Experimental Economics Results, 1, 138-145.

Dormady, N. (2016): "Carbon Auction Revenue and Market Power: An Experimental Analysis," Energies, $9,897$.

Dormady, N. C. (2014): "Carbon auctions, energy markets \& market power: An experimental analysis," Energy Economics, 44, 468-482.

FAbra, N., N.-H. Fehr, AND D. HARBord (2006): "Designing electricity auctions," The RAND Journal of Economics, 37, 23-46. 
FischBACHER, U. (2007): "z-Tree: Zurich toolbox for ready-made economic experiments," Experimental economics, 10, 171-178.

Genc, T. S. And S. S. Reynolds (2011): "Supply function equilibria with capacity constraints and pivotal suppliers," International Journal of Industrial Organization, 29, 432-442.

Green, R. (1996): "Increasing competition in the British electricity spot market," The Journal of Industrial Economics, 205-216.

Greene, William, H. (2012): Econometric Analysis, Prentice Hall.

Greiner, B. (2004): "An Online Recruitment System for Economic Experiments," in Forschung und wissenschaftliches Rechnen, Goettingen: GWD.

Heftr, A. (2017): "Equilibria in symmetric games: Theory and Applications," Theoretical Economics, 12.

Hefti, A. AND P. SHEn (2019): "Supply function competition with asymmetric costs: Theory and experiment," Economics Letters 178.

Holmberg, P. (2008): "Unique supply function equilibrium with capacity constraints," Energy Economics, $30,148-172$.

Holmberg, P., D. Newbery, AND D. Ralph (2013): "Supply function equilibria: Step functions and continuous representations," Journal of Economic Theory, 148, 1509-1551.

HolmberG, P. And F. Wolak (2015): "Electricity markets: Designing auctions where suppliers have uncertain costs," Working Paper.

Holt, C. A. (1989): "The exercise of market power in laboratory experiments," The Journal of Law \& Economics, 32, 107-130.

Hortaçsu, A., J. Kastl, And A. Zhang (2018): "Bid shading and bidder surplus in the us treasury auction system," American Economic Review, 108, 147-69.

HortaÇSu, A. AND D. MCADAms (2010): "Mechanism choice and strategic bidding in divisible good auctions: An empirical analysis of the turkish treasury auction market," Journal of Political Economy, $118,833-865$. $157-84$

(2018): "Empirical work on auctions of multiple objects," Journal of Economic Literature, 56,

Hortaçsu, A. And S. L. Puller (2008): "Understanding strategic bidding in multi-unit auctions: a case study of the Texas electricity spot market," The RAND Journal of Economics, 39, 86-114.

Huck, S., H.-T. Normann, And J. Oechssler (2000): "Does information about competitors' actions increase or decrease competition in experimental oligopoly markets?" International Journal of Industrial Organization, 18, 39-57.

(2004): "Two are few and four are many: number effects in experimental oligopolies," Journal of Economic Behavior \& Organization, 53, 435-446.

Joskow, P. AND E. KAHN (2002): "A quantitative analysis of pricing behavior in California's wholesale electricity market during Summer 2000," Energy Journal, 23, 1-35.

Kastl, J. (2011): "Discrete bids and empirical inference in divisible good auctions," The Review of Economic Studies, 78, 974-1014.

KIM, J. AND Y.-K. CHE (2004): "Asymmetric information about rivals' types in standard auctions," Games and Economic Behavior, 46, 383-397.

Klemperer, P. D. And M. A. Meyer (1989): "Supply function equilibria in oligopoly under uncertainty," Econometrica, 1243-1277.

Kwasnica, A. M. And K. Sherstyuk (2013): "Multiunit auctions," Journal of economic surveys, 27, 461-490.

Malvey, P. F. And C. M. Archibald (1998): "Uniform-price auctions: Update of the Treasury experience," US Treasury.

Milgrom, P. R. And R. J. Weber (1982): "A theory of auctions and competitive bidding," Econometrica, 1089-1122. 
Morath, F. AND J. Münster (2008): "Private versus complete information in auctions," Economics Letters, 101, 214-216.

Ockenfels, A. (2007): "Stromboerse und Marktmacht," Energiewirtschaftliche Tagesfragen, 57, 44-58.

Parente, P. M. And J. M. S. Silva (2016): "Quantile regression with clustered data," Journal of Econometric Methods, 5, 1-15.

PERRY, M. AND P. J. RENy (1999): "On the Failure of the Linkage Principle in multi-unit auctions," Econometrica, 67, 895-900.

Pesendorfer, M. (2000): "A study of collusion in first-price auctions," The Review of Economic Studies, $67,381-411$.

Ranaldo, A. AND E. Rossi (2016): "Uniform-price auctions for Swiss government bonds: Origin and evolution," University of St. Gallen, School of Finance Research Paper.

Rassenti, S. J., V. L. Smith, And B. J. Wilson (2003): "Controlling market power and price spikes in electricity networks: Demand-side bidding," Proceedings of the National Academy of Sciences, 100 , 2998-3003.

Stoft, S. (2002): Power System Economics, IEEE Press and Wiley-Interscience.

TiRole, J. (1988): The theory of industrial organization, MIT press.

Vives, X. (1999): Oligopoly pricing, Cambridge MIT Press. 1966.

(2011): "Strategic supply function competition with private information," Econometrica, 79, 1919-

VON DER FEHR, N.-H. M. (2013): “Transparency in electricity markets," Economics of Energy E Environmental Policy, 2, 87-105.

WolAK, F. A. (2003): "Measuring unilateral market power in wholesale electricity markets: the California market, 1998-2000," The American economic review, 93, 425-430.

Wolfram, C. D. (1998): "Strategic Bidding in a Multiunit Auction: An Empirical Analysis of Bids to Supply Electricity in England and Wales," The Rand Journal of Economics, 703-725. 


\section{A Proofs}

Proof Lemma 1 Clearly, $\sum_{j} q_{j}(P)<\bar{Q}$ cannot be a SFE, as then $P^{*}=P_{\max }$, and any unconstrained bidder could marginally increase her quantity without reducing the price. Further, $\sum_{j} q_{j}(P)>\bar{Q}$ is not possible in any SFE. To see why, note that if $\sum_{j} q_{j}(P)>\bar{Q}$ we either have that $\sum_{j} q_{j}\left(P^{\prime}\right)=\bar{Q}$ for some $P^{\prime} \in[0, P)$, or $\sum_{j} q_{j}(0)>\bar{Q}$. In the former, $P$ cannot occur in a SFE as the auction mechanism would select $P^{\prime}$ instead, while in the later the equilibrium price is $P=0$, which implies a loss for all bidders with $q_{j}(0)>0$. The claim about symmetric SFE then is obvious.

Proof Theorem 1 a) "If". Let $P \in\left(c, P_{\max }\right]$. We need to show that we can find $q \in \mathcal{F}$ such that $(2)$ is satisfied. By $(2),(P, q(P)), q(P) \in \mathcal{F}$, is a SymSFE iff $q(P)=\frac{\bar{Q}}{m+1}$ and

$$
q^{\prime}(P)=\frac{\bar{Q}}{m(m+1)(P-c)}>0
$$

by (2). As these two are the only equilibrium requirements, any $q \in \mathcal{F}$ that complies with them forms a SymSFE.

"Only if" Suppose that $P \leq c$. Obviously, $P<c$ can never be part of any symSFE because such a price would entail a loss. Hence suppose that $P=c$ is part of a symSFE, thus $q(c)=\frac{\bar{Q}}{m+1}>0$ and $q^{\prime}(c)>0$. Then, an individual firm $j$ could deviate by marginal bid shading at $P=c$ and earn a positive payoff. In particular, the continuity of $q(P)$ assures that there is a $P>c$ such that $0<q_{j}(P)<\frac{\bar{Q}}{m+1} .55$ The remaining claims then follow directly from $(29)$ and the fact that $\Pi\left(P^{*}\right)=\frac{\bar{Q}}{m+1}\left(P^{*}-c\right)$.

b) We show that given any symSFE $\left(P^{*}, q(P)\right)$ a LinSFE can be constructed from it. Let $P^{*} \in\left(c, P_{\max }\right]$ be part of a symSFE, and set $\beta^{*}=q^{\prime}\left(P^{*}\right)>0$ in $(29)$, which implies that

$$
P^{*}-c=\frac{\bar{Q}}{m(m+1) \beta^{*}}
$$

Using this in $\alpha^{*}+\beta^{*} P^{*}=\frac{\bar{Q}}{m+1}$ yields $(3)$.

Proof Corollary 1 Note that (4) is equivalent to (30), and the first claim follows because $\beta^{*}=q^{\prime}\left(P^{*}\right)>0$ and $\alpha^{*}+\beta^{*} P$ is the first-order Taylor polynomial of $q(P)$ evaluated at $P^{*}$. Further, (5) follows from (4), because $\Pi^{*}=\frac{\bar{Q}}{m+1}\left(P^{*}-c\right)$, and $P^{*}>c$ because $\beta^{*}>0$ by Theorem 1. Because in any symSFE equilibrium profits are determined only by $P^{*}$, any possible symSFE

\footnotetext{
${ }^{55}$ While $c$ cannot be supported as a symSFE with a differentiable $q(P)$ as required by Definition $2, P=c$ could be a symmetric SFE if one considers more general supply functions.
} 
with equilibrium price $P^{*}$ generate the same level of equilibrium profits.

Proof Proposition 1 Let $m=n-1$. By pivotality, a single bidder can always shade bids such that $P^{*}=P_{\max }$ results. By playing such a strategy, the bidder can assure herself at least the minimal pivotal payoff $(\bar{Q}-m \bar{k})\left(P_{\max }-c\right)$. Now, suppose that $c<P^{*}<P_{\max }$ is the equilibrium price of a SFE, hence $\sum_{j} q_{j}\left(P^{*}\right)=\bar{Q}$. In any such SFE the max-min payoff is $\frac{\bar{Q}}{m+1}\left(P^{*}-c\right)$. For $P^{*}$ to be an equilibrium price, this payoff cannot be below the minimal pivotal payoff, which implies that

$$
P^{*} \geq c+(m+1)\left(P_{\max }-c\right) \frac{\bar{Q}-m \bar{k}}{\bar{Q}} \equiv \hat{P} .
$$

Hence any $P<\hat{P}$ cannot be part of a SFE which, together with the observation that $c<\hat{P}<P_{\max }$, proves the first claim. Given that all bidders are symmetric, Theorem 1 and Corollary 1 both apply to the price range $\left[\hat{P}, P_{\max }\right]$, meaning that restriction (3) and conditions (4), (5) apply, proving the second claim.

Proof Proposition 2 By pivotality, the low-cost bidder can assure herself a quantity of $q_{L}(100)=$ 100 , and thus a payoff $\Pi_{L}=8200$. For $P^{*}<100$ to be supported as a SFE, the low-cost bidder needs at least this payoff. The most favorable condition is where the low-cost bidder can supply the full 120 units. Direct calculation shows that for this to exceed $\Pi=8200$, a price above 91.67 is needed, which proves the first claim. Given $\Delta=+5$, the only deviation candidate is $P^{*}=95$, so suppose that indeed $P^{*}=95$ is a SFE with $q_{L}^{*}=120$. Thus the two high-cost bidders jointly sell 60 . Note that each high-cost seller is likewise pivotal, and cannot sell less than 20 units. We now claim the the high-cost seller with the smallest supposed equilibrium quantity $q_{H}^{*}$ always has an incentive to deviate to the price cap. Indeed, this bidder could marginally reduce her quantity, exploiting her pivotality to push the price to $1 \overline{0} 0$; the price jump of 5 always compensates such a small loss of quantity, which shows that $P^{*}=95$ cannot be supported as a SFE. A simple and intuitive example that supports the $P^{*}=100$ as a SFE is where both high-cost bidders set $q_{H}(P)=40$ for any $P>\bar{c}$, while the low-cost bidder completely shades his bids up to $P=100$, where he sets $q_{1}(100)=120$.

Proof Proposition 3 The proof builds on the following insight.

Lemma 2 Let $j=2,3$ indicate the two high-cost bidders in situation $\left(s_{3}, 100\right)$. If the high-cost supply function profile is such that $q_{2}(\bar{c})+q_{3}(\bar{c})<20$, then seizing the market at $P=\bar{c}$ is never optimal for the low-cost firm. 
Proof: A strategy with $q_{L}(\bar{c}) \geq 100$ assures the single low-cost bidder a payoff $80(\bar{c}-\underline{c})=3200$. Given the profile of the two high-cost types, the low-cost bidder can alternatively choose to shade bids to achieve $P^{*}>\bar{c}$. By pro-rata rationing and given the stated high-cost profiles he can always assure himself at least $q_{L}=80$ in any such equilibrium. The worst possible outcome payoff of such a strategy for the low-cost bidder then occurs if $P^{*}=55$, in which case he still earns a payoff of $45 * 80=3600$. Hence seizing the market at $P^{*}=\bar{c}$ cannot be optimal.

We now prove Proposition 3. If all bidders play the stated strategy, the market clearing price is $P^{*}=\bar{c}+\Delta$, and the low-cost bidder sells more than $q_{L}=80$ by the pro-rata rationing rule, earning a payoff of more than $80(\bar{c}+\Delta-\underline{c})=3600$. The possible deviations of the low-cost bidder are i) either to capture the market at $P=\bar{c}$ or ii) to shade bids beyond $P=\bar{c}+\Delta$, which implies higher marginal costs. Neither of these strategies is a profitable deviation, as we show next. First, market capturing $q_{L}(\bar{c}) \geq 100$ fails to be a profitable deviation by Lemma 2. Second, the most favorable situations for the low-cost bidder, given the stated strategy profile of the high-cost bidders, is to shade bids up to $P=100$. This yields a payoff $\hat{\Pi}=(100-80) * 90=1800<3600$, showing that the deviation is not profitable. In turn, high-cost bidders can make positive profits only if $P^{*}>\bar{c}$. Given the stated supply function $q_{L}$ it thus is a best response of the high-cost bidders to bid their full capacity already at $P=\bar{c}+\Delta$.

Proof Theorem 2 Note first that $\frac{\bar{m}}{m+1}>2 \mu \bar{m}-1$ implies $1>\bar{m} \mu$. "If". Suppose that $\beta^{*}>0$, and $\left(\alpha^{*}, \beta^{*}\right)$ are such that condition (12) holds. Solving (12) for $\alpha^{*}$ and using this in (11) yields (14). This price $P^{*}$ is uniquely determined given $\left(\alpha^{*}, \beta^{*}\right)$, and $P^{*}>c$ follows from $1>\mu \bar{m}$. As $P^{*}$ yields a strictly positive payoff, $P^{*}$ in (11) indeed maximizes (10). As the aggregate supply function is $(m+1)\left(\alpha^{*}+\beta^{*} P\right)$, condition (12) together with the state-wise market clearing implies that the state-wise prices $P_{m}^{*}$ are

$$
P_{m}^{*}=\min \left\{c+\frac{\bar{Q}}{\beta} \frac{\bar{m}-(m+1)(2 \mu \bar{m}-1)}{\bar{m}(m+1)}, P_{\max }\right\} .
$$

We claim that $E\left[P_{m}^{*}\right]=P^{*}$ as a consequence of (13). Let $\hat{P}_{m} \equiv c+\frac{\bar{Q}}{\beta} \frac{\bar{m}-(m+1)(2 \mu \bar{m}-1)}{\bar{m}(m+1)}$ in (31), and note that $\hat{P}_{m}$ is strictly decreasing in $m$ and $E\left[\hat{P}_{m}\right]=P^{*}$. It is easy to verify that $P_{m}^{*}=\hat{P}_{m}$ for $m \geq 0$ if and only if (13) holds. Thus (13) immediately assures that $E\left[P_{m}^{*}\right]=P^{*}$, and (9) holds because all state-wise markets clear. Thus all requirements of an A-LinSFE are met.

"Only if". First, $\beta^{*}=0$ is impossible in an A-LinSFE because of (11). Hence $\beta^{*}>0$ in any A-LinSFE. By presumption, $\bar{P}=P^{*}$ and (9) hold, hence also $\alpha^{*}+\beta^{*} P^{*}=\bar{Q} \mu$. This equation 
together with (11) implies (12). Therefore, $P_{m}^{*}$ must be given by (31). Moreover, the presumption that $E\left[P_{m}^{*}\right]=P^{*}$ implies (13). For if (13) is violated by some $\beta^{*}$, then this value of $\beta^{*}$ implies $P_{0}^{*}=P_{\max }<\hat{P}_{0}$ and $P_{m}^{*} \leq \hat{P}_{m}$, which implies $E\left[P_{m}^{*}\right]<E\left[\hat{P}_{m}\right]=P^{*}$, contradiction.

For the remaining claims, note that the range of average prices (15) follows from (14), $\beta^{*}>0$ and (13). Finally, (16) follows from (31) and the fact that $\hat{P}_{m}=P_{m}^{*}$, and $P_{m}^{*}>c$ follows from $\frac{\bar{m}}{m+1}>2 \mu \bar{m}-1$.

Proof Corollary 3 The first claim follows, as by the proof of Theorem 2 we have $P_{m}^{*}=\hat{P}_{m}$, where the latter is strictly decreasing in $m$. Next, the claimed monotonicity in $\bar{Q}$ can be directly verified from (14) and (16). Finally, the least competitive bidding is the one where $\beta^{*}$ is as small as possible, which requires that inequality (13) binds. Then, (16) implies that $P_{0}^{*}=P_{\max }$.

Proof Theorem 3 The proof essentially mimicks the one from Theorem 2. "If" Suppose that $\beta^{*}>0$, and $\left(\alpha^{*}, \beta^{*}\right)$ are such that condition (22) holds. Solving (22) for $\alpha^{*}$ and using this in (19) yields (24). This price $P^{*}$ is uniquely determined given $\left(\alpha^{*}, \beta^{*}\right)$. As $P^{*}$ yields a strictly positive payoff $(18), P^{*}$ in (24) indeed maximizes (18). As the aggregate supply function is $(m+1)\left(\alpha^{*}+\right.$ $\left.\beta^{*} P\right)$, condition $(22)$ together with the state-wise market clearing implies that the state-wise prices $P_{\bar{Q}}^{*}$ are

$$
P_{\bar{Q}}^{*}=\min \left\{c+\frac{m \bar{Q}-(m-1) \Gamma}{\beta^{*} m(m+1)}, P_{\max }\right\}
$$

We claim that $E\left[P_{\bar{Q}}^{*}\right]=P^{*}$ as a consequence of $(23)$. Let $\hat{P}_{\bar{Q}} \equiv c+\frac{m \bar{Q}-(m-1) \Gamma}{\beta^{*} m(m+1)}$ in (32), and note that $\hat{P}_{\bar{Q}}$ is strictly increasing in $\bar{Q}$ and $E\left[\hat{P}_{\bar{Q}}\right]=P^{*}$. It is easy to verify that $P_{\bar{Q}}^{*}=\hat{P}_{\bar{Q}}, \forall \bar{Q}$, if and only if (23) holds. Thus (23) immediately assures that $E\left[P_{\bar{Q}}^{*}\right]=P^{*}$, and (21) holds because all state-wise markets clear. Thus all requirements of an A-LinSFE are met.

"Only if" First, $\beta^{*}>0$ in any A-LinSFE as a consequence of (19). By presumption, $\bar{P}=P^{*}$ and (21) hold, hence also $\alpha^{*}+\beta^{*} P^{*}=\frac{\Gamma}{m+1}$. This equation together with (19) implies (22). Therefore, $P_{\bar{Q}}^{*}$ must be given by (32). Moreover, the presumption that $E\left[P_{\bar{Q}}^{*}\right]=P^{*}$ implies (23). For if (23) is violated by some $\beta^{*}$, then this value of $\beta^{*}$ implies $P_{\bar{Q}_{v}}^{*}=P_{\max }<\hat{P}_{\bar{Q}_{v}}$ and $P_{\bar{Q}}^{*} \leq \hat{P}_{\bar{Q}}$, which implies $E\left[P_{\bar{Q}}^{*}\right]<E\left[\hat{P}_{\bar{Q}}\right]=P^{*}$, contradiction.

For the remaining claims, note that the range of average prices (25) follows from $(24), \beta^{*}>0$ and (23). Finally, (26) follows from (32) and the fact that $\hat{P}_{\bar{Q}}=P_{\bar{Q}}^{*}$, and $P_{\bar{Q}}^{*}>c$ follows from $m \bar{Q}_{1}-(m-1) \Gamma>0$, which implies a positive payoff in each state.

Proof Corollary 4 The first claim holds, as by the proof of Theorem 3 we have $P_{\bar{Q}}^{*}=\hat{P}_{\bar{Q}}$, where the latter is strictly increasing in $\bar{Q}$. Next, the claimed monotonicity in $m$ can be directly 
verified by differentiating (24) and (26) accordingly. Finally, the least competitive bidding is the one where $\beta^{*}$ is as small as possible, which requires that inequality (23) binds. Then, (26) implies that $P_{\bar{Q}_{v}}^{*}=P_{\max }$.

Proof Proposition 4 Given the stated high-cost supply functions, (28) is a best response for any possible realization of $\bar{Q}$. This follows directly from the fact that the low-cost bidder wishes to seize the entire market in case of exclusive market power (Hefti and Shen, 2019; Prop. 1), and from Propositions 2 and 3. Offering the full capacity at $P>\bar{c}$ is a best response of the high-cost bidders to $(28)$.

\section{B Further results}

\section{B.1 Inexistence of linear symmetric (Bayesian) SFE with supply uncertainty}

Suppose that $\bar{q}(P)$ is a $C^{2}$-function. Maximizing (7) for a given value of $m$ then yields the first-order condition

$$
q(P(m))=m \bar{q}^{\prime}(P(m))(P(m)-c)
$$

Using $q(P)=\alpha+\beta P$ and market clearing (8) in this equation yields $\frac{\bar{Q}}{m+1}=m \beta(P(m)-c)$, or

$$
P(m)=c+\frac{\bar{Q}}{m(m+1) \beta}, \quad \forall m
$$

At the same time, we need that $\alpha+\beta P(m)=\frac{\bar{Q}}{m+1}$ for each $m$. Jointly, with the last equation, this yields

$$
\alpha+\beta c=\frac{\bar{Q}(m-1)}{m(m+1)}, \quad \forall m
$$

which is impossible if $\alpha, \beta$ are constant. ${ }^{56}$

\section{B.2 Pro-rata protocol}

Let $\mathcal{A} \equiv\left\{P \in\left[0, P_{\max }\right]: q(P) \geq \bar{Q}\right\}$, and $\hat{q}_{j}\left(P_{0}\right)=\sup \left\{q_{j}(P): q_{j}(P)<q_{j}\left(P_{0}\right)\right\}$. The auction price $P^{*}$ either is the lowest price for which $q(P) \geq \bar{Q}$, or the price cap: $P^{*}=\inf \mathcal{A}$ if $q\left(P_{\max }\right) \geq \bar{Q}$; $P^{*}=P_{\max }$ else. Given $P^{*}$, the pro-rata auction allocation $\left(q_{1}^{*}, \ldots, q_{n}^{*}\right)$ is: If $q\left(P_{\max }\right)<\bar{Q}$ then $q_{j}^{*}=q_{j}\left(P_{\max }\right)$; otherwise if $q\left(P^{*}\right) \geq \bar{Q}$ then i) $q_{j}^{*}=\hat{q}_{j}\left(P^{*}\right)$ if $\sum_{j} \hat{q}_{j}\left(P^{*}\right)=\bar{Q}$, and ii) $q_{j}^{*}=$ $\hat{q}_{j}\left(P^{*}\right)+\frac{q_{j}\left(P^{*}\right)}{\sum_{j} q_{j}\left(P^{*}\right)}\left(\bar{Q}-\sum_{j} \hat{q}_{j}\left(P^{*}\right)\right)$ if $\sum_{j} \hat{q}_{j}\left(P^{*}\right)<\bar{Q}$.

\footnotetext{
${ }^{56}$ While globally linear symmetric Bayesian SFE fail to exist, one could principally seek to derive Bayesian SFE which are piecewise linear, e.g., using constructions similar to those Baldick et al. (2004) or Genc and Reynolds (2011) invoke in case of demand uncertainty.
} 


\section{B.3 CLS estimation in uncertainty treatments}

The top parts of Tables 9 and 10 show the CLS estimates in case of supply and demand uncertainty, respectively. The middle parts of these tables display the corresponding price predictions, $\hat{P}_{C L S}$, jointly with their unrestricted OLS counterparts $\hat{P}_{O L S}$, and the corresponding true average prices $P^{*}$. With supply uncertainty, $P^{*}$ is the true average price pooled across competition states $s_{1}-s_{3}$. A comparison of $\hat{P}_{C L S}, P^{*}$ and $\hat{P}_{O L S}$ shows, similar to the full information case, that the CLS price prediction is statistically close to the true average price, and tends to outperform the OLS price prediction in this respect. With demand uncertainty, $P^{*}$ is the true average price pooled across demand levels $\bar{Q}=60,100,180$ for state $s_{2}$, and $\bar{Q}=60,100$ for state $s_{4}$ (as $\bar{Q}=180$ is degenerate in $s_{4}$ due to the design). Again, the CLS price predictions are closer to the true average prices than their OLS counterparts.

Any given A-LinSFE uniquely determines all state-wise prices in each of the two uncertainty treatments (see (16) and (26)). Hence a comparison between the state-wise CLS price predictions with the corresponding true average prices delivers an additional consistency check for whether A-LinSFE yields reasonable predictions about the average equilibrium outcomes. Specifically, if average bidding corresponds to an A-LinSFE, then the related state-wise average prices should sit on the same linear supply function (see Figure 3 ). The necessary estimation results are summarized in the bottom parts of Tables 9 and 10. For supply uncertainty, the three possible states are $s=s_{1}, s_{2}, s_{3}$. In all three states, we cannot reject the null that the state-wise CLS prices, $\hat{P}_{s}$, and the true average prices, $P_{s}^{*}$, are the same (last row of Table 9). Likewise, we cannot reject that the predicted CLS prices in the various demand states are the same as the corresponding true average prices in case of demand uncertainty. Moreover, we compared the state-wise prices predicted by standard OLS (not reported) to their CLS and true average counterparts. We found the CLS predictions to be closer to the true average prices than their OLS counterparts in all cases.

Next, we verify whether the CLS estimates of $\hat{\beta}^{*}$ are consistent with the admissible values of $\beta$ in A-LinSFE. The top part of Tables 9 and 10 contain the range of $\beta$ identified by (17) in case of supply uncertainty, and by (27) with demand uncertainty. ${ }^{57}$ With one exception, all estimated values of $\hat{\beta}^{*}$ reside in the corresponding interval. In case of $s_{2}$ and demand uncertainty, the value $\hat{\beta}=1.94$ is slightly below the lower bound $\underline{\beta}=2$. However, this difference is not significant ( $p$-value 0.49 for the t-test that $\hat{\beta}=2$ ). Hence we conclude that the estimated $\hat{\beta}^{*}$ are consistent with the admissible values identified by A-LinSFE.

\footnotetext{
${ }^{57}$ The intervals $[\beta, \bar{\beta}]$ are obtained from (17) with parameter values $\mu=5 / 12, \bar{m}=1, c=10, \bar{P}=55$ and $\bar{Q} \in\{60,80\}$. We used $\bar{Q}=80$ instead of $\bar{Q}=100$ because low-cost bidders aim at maximally supplying 80 units in this market situation given their increase in marginal costs.
} 
Table 9: CLS Supply function estimation: Supply uncertainty

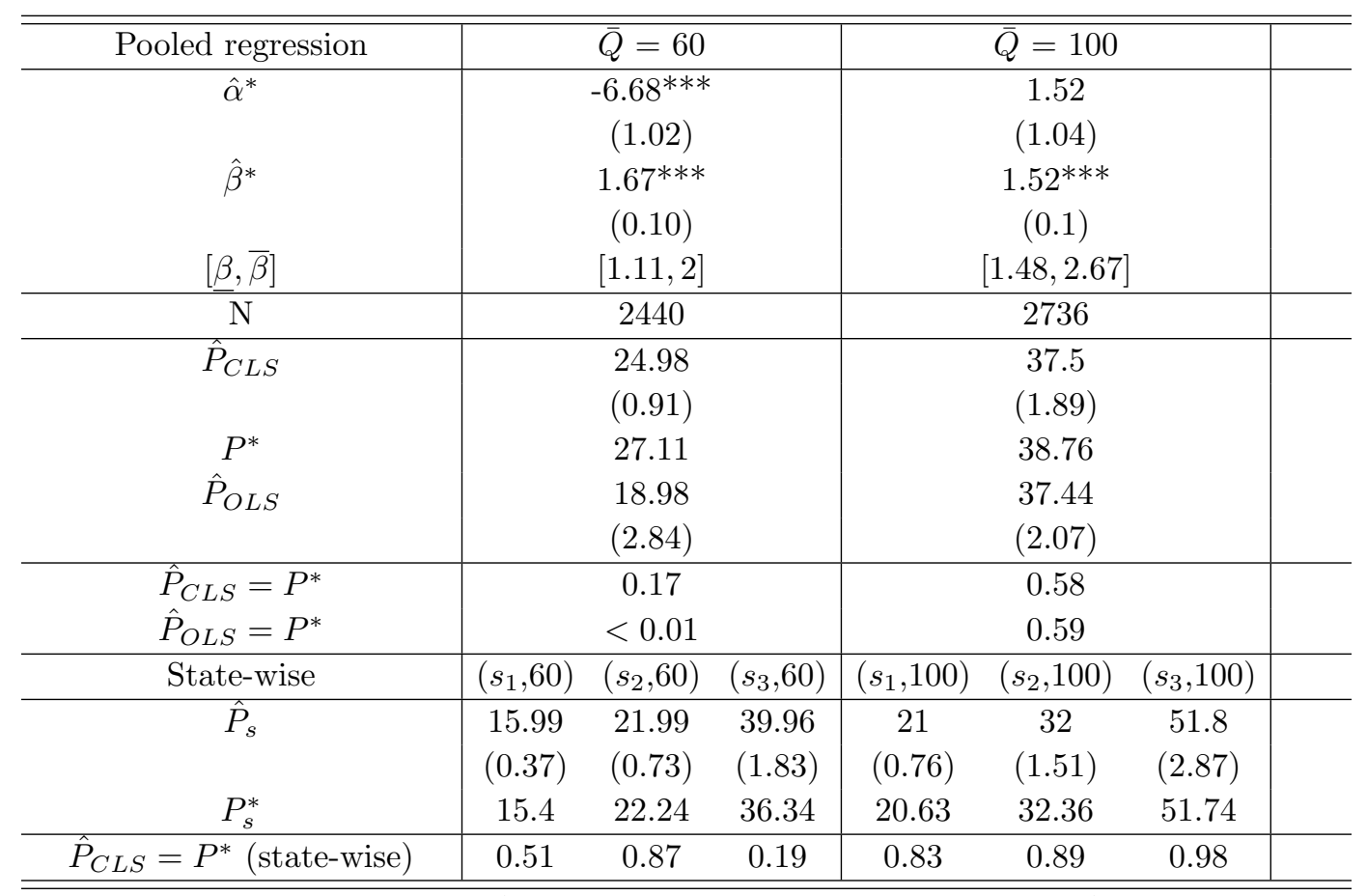

Unit of observation: Subject. $\hat{\alpha}^{*}$ and $\hat{\beta}^{*}$ are estimated parameters of A-LinSFE, and $\hat{P}_{C L S}$ is the predicted price by the CLS estimates, with clustered SE in parentheses. Restriction (12) was used, where the data was pooled over states $s_{1}-s_{3} . \hat{P}_{O L S}$ is the predicted price by the OLS estimates of $\alpha+\beta P$ for the same data. $P^{*}$ is the true average price pooled over $s_{1}-s_{3} .[\beta, \bar{\beta}]$ is the range of admissible values for $\beta$ in A-LinSFE obtained from (17). $\hat{P}_{s}$ and $P_{s}^{*}$ indicate the state-wise CLS prediction and the true average state-wise price, respectively. Significance level: ${ }^{* * *} p<.01$. 
Table 10: CLS Supply function estimation: Demand uncertainty

\begin{tabular}{|c|c|c|c|c|}
\hline Pooled regression & \multicolumn{3}{|c|}{$s_{2}$} & $s_{4}$ \\
\hline$\hat{\alpha}^{*}$ & \multicolumn{3}{|c|}{$-19.43^{* * *}$} & $\begin{array}{c}-22.43^{* * *} \\
(3.17)\end{array}$ \\
\hline$\hat{\beta}^{*}$ & \multirow{2}{*}{\multicolumn{3}{|c|}{$1.94 * * *$}} & $0.72^{* * *}$ \\
\hline & & & & $(0.06)$ \\
\hline$[\underline{\beta, \bar{\beta}}]$ & \multicolumn{3}{|c|}{$\begin{array}{c}(0.08) \\
{[2.6]}\end{array}$} & {$[0.4,1.33]$} \\
\hline $\mathrm{N}$ & \multicolumn{3}{|c|}{3960} & 1080 \\
\hline$\hat{P}_{C L S}$ & \multicolumn{3}{|c|}{$\begin{array}{l}39.16 \\
(1.23)\end{array}$} & $\begin{array}{l}68.64 \\
(1.65)\end{array}$ \\
\hline$P^{*}$ & \multicolumn{3}{|c|}{39.16} & 70.38 \\
\hline$\hat{P}_{O L S}$ & \multicolumn{3}{|c|}{37.40} & 67.07 \\
\hline & \multicolumn{3}{|c|}{$(1.88)$} & $(3.26)$ \\
\hline$\hat{P}_{C L S}=P^{*}$ & \multirow{2}{*}{\multicolumn{3}{|c|}{0.50}} & 0.54 \\
\hline$\hat{P}_{O L S}=P^{*}$ & & & & 0.41 \\
\hline Demand-wise & $\left(s_{2}, 60\right)$ & $\left(s_{2}, 100\right)$ & $\left(s_{2}, 180\right)$ & $\left(s_{4}, 60\right) \quad\left(s_{4}, 100\right)$ \\
\hline \multirow[t]{2}{*}{$\hat{P}_{\bar{Q}}$} & 25.44 & 35.73 & 56.32 & $59.32 \quad 77.97$ \\
\hline & $(0.65)$ & $(1.09)$ & $(1.95)$ & $(2.48)$ \\
\hline$P_{\bar{Q}}^{*}$ & 24.5 & 33.73 & 59.17 & 61.05 \\
\hline$\hat{P}_{C L S}=P^{*}$ (demand-wise) & 0.68 & 0.47 & 0.40 & 0.44 \\
\hline
\end{tabular}

Unit of observation: Subject. $\hat{\alpha}^{*}$ and $\hat{\beta}^{*}$ are estimated parameters of A-LinSFE, and $\hat{P}_{C L S}$ is the predicted price by the CLS estimates, with clustered SE in parentheses. Restriction (22) was used, where the data was pooled over demand levels $60-180 . \hat{P}_{O L S}$ is the predicted price by the OLS estimates of $\alpha+\beta P$ for the same data. $P^{*}$ is the true average price pooled over $60-180 .[\beta, \bar{\beta}]$ is the range of admissible values for $\beta$ in A-LinSFE obtained from (27). The demand-wise CLS prediction and the corresponding true average prices are indicated by $\hat{P}_{s}$ and $P_{s}^{*}$, respectively. Significance level: ${ }^{* * *} p<.01$.

Table 11: $\beta$ : Comparative-statics

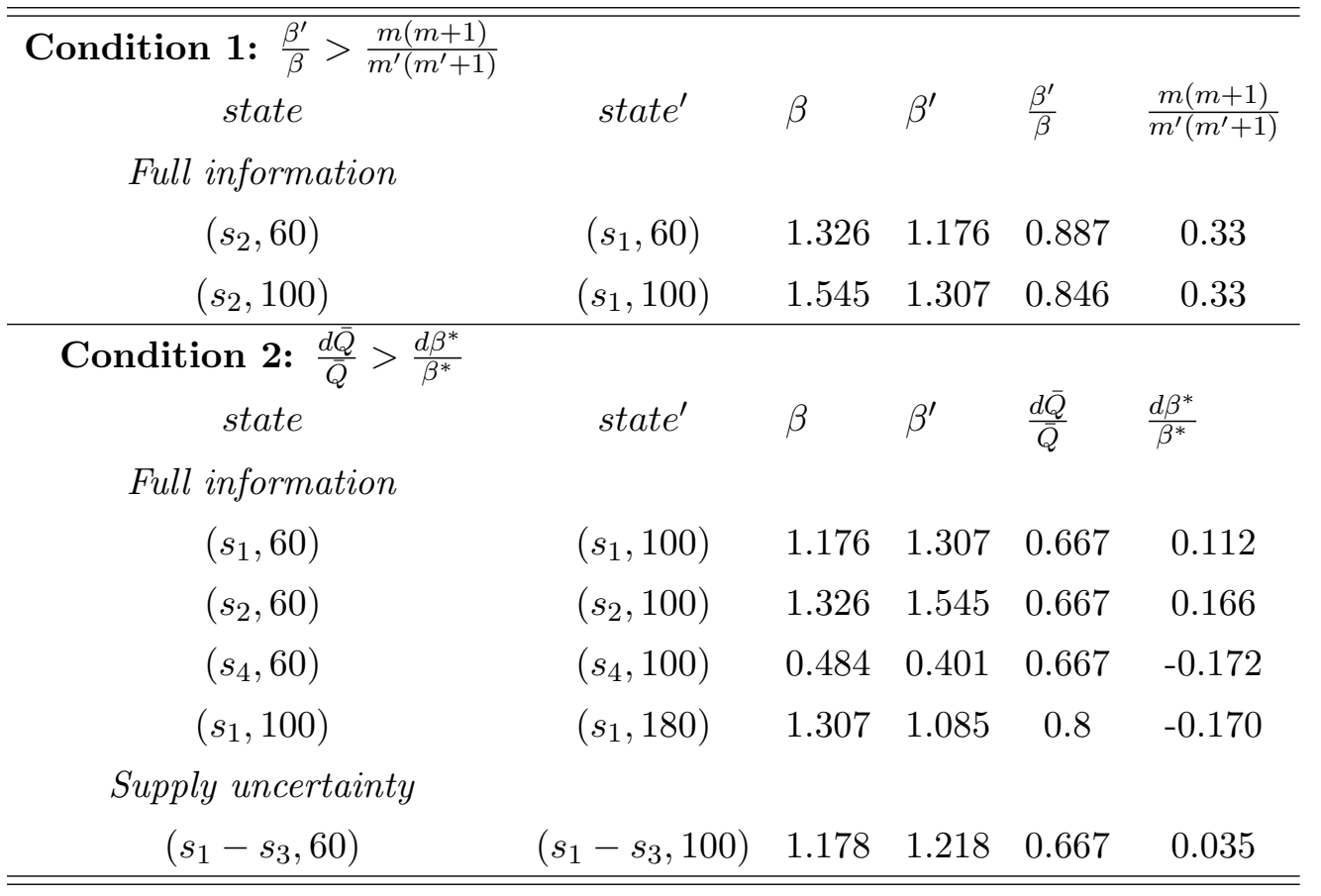

The table summarizes the estimated values of $\beta$ and the comparative-static requirements for competition and demand effects as identified by Corollaries $2-3$. 
Finally, Table 11 shows that the estimated values of $\beta$, as $m$ and $\bar{Q}$ change, are consistent with the comparative-static requirements identified by Corollaries 2 and 3, given that we empirically found the true average prices to significantly increase in $\bar{Q}$ and decrease in $m$.

\section{B.4 Data stratification}

Table 12: Data stratification

\begin{tabular}{|c|c|c|c|c|}
\hline \multicolumn{5}{|c|}{ Panel A: $\bar{Q}=60$} \\
\hline & $s_{1}$ & $s_{2}$ & $s_{3}$ & $s_{4}$ \\
\hline$T_{B}$ & $\begin{array}{c}P<10 ; P>60 \\
7.10 \%\end{array}$ & $\begin{array}{c}P<10 ; P>60 \\
4.80 \%\end{array}$ & $\begin{array}{c}P<=20 ; P>55 \\
11.90 \%\end{array}$ & $\begin{array}{c}P<40 ; P>90 \\
3.60 \%\end{array}$ \\
\hline$T_{D}$ & $\begin{array}{c}P<10 \\
5 \%\end{array}$ & None & $\begin{array}{l}P>55 \\
6.70 \%\end{array}$ & $\begin{array}{c}P>=90 \\
5 \%\end{array}$ \\
\hline$T_{S}$ & $\begin{array}{c}P<10 ; P>30 \\
7.40 \%\end{array}$ & $\begin{array}{c}P<10 ; P>45 \\
10.70 \%\end{array}$ & $\begin{array}{c}P<10 ; P>55 \\
9.50 \%\end{array}$ & $\begin{array}{c}P<50 ; P>80 \\
4 \%\end{array}$ \\
\hline \multicolumn{5}{|c|}{ Panel B: $\bar{Q}=100$} \\
\hline & $s_{1}$ & $s_{2}$ & $s_{3}$ & $s_{4}$ \\
\hline$T_{B}$ & $\begin{array}{c}P<10 ; P>45 \\
10.70 \%\end{array}$ & $\begin{array}{c}P<10 ; P>60 \\
3.60 \%\end{array}$ & $\begin{array}{c}P<=25 \\
3.60 \%\end{array}$ & None \\
\hline$T_{D}$ & $\begin{array}{c}P<10 ; P>60 \\
5 \%\end{array}$ & $\begin{array}{c}P=100 \\
1.60 \%\end{array}$ & $\begin{array}{l}P<20 \\
1.70 \%\end{array}$ & None \\
\hline$T_{S}$ & $\begin{array}{c}P<10 ; P>45 \\
7.70 \%\end{array}$ & $\begin{array}{c}P<10 ; P>55 \\
5.30 \%\end{array}$ & $\begin{array}{l}P>70 \\
6.80 \%\end{array}$ & None \\
\hline \multicolumn{5}{|c|}{ Panel C: $\bar{Q}=180$} \\
\hline & $s_{1}$ & $s_{2}$ & $s_{3}$ & $s_{4}$ \\
\hline$T_{B}$ & None & None & None & None \\
\hline$T_{D}$ & $\begin{array}{c}P<10 ; P>65 \\
5 \%\end{array}$ & None & None & None \\
\hline$T_{S}$ & $\begin{array}{c}P<10 ; P>55 \\
16.70 \%\end{array}$ & $\begin{array}{c}P<=15 \\
1.40 \%\end{array}$ & None & None \\
\hline
\end{tabular}

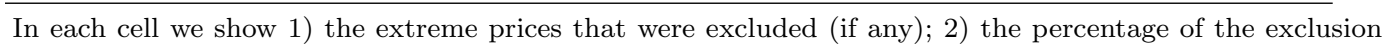
for each cell. 


\section{B.5 Practitioners and students}
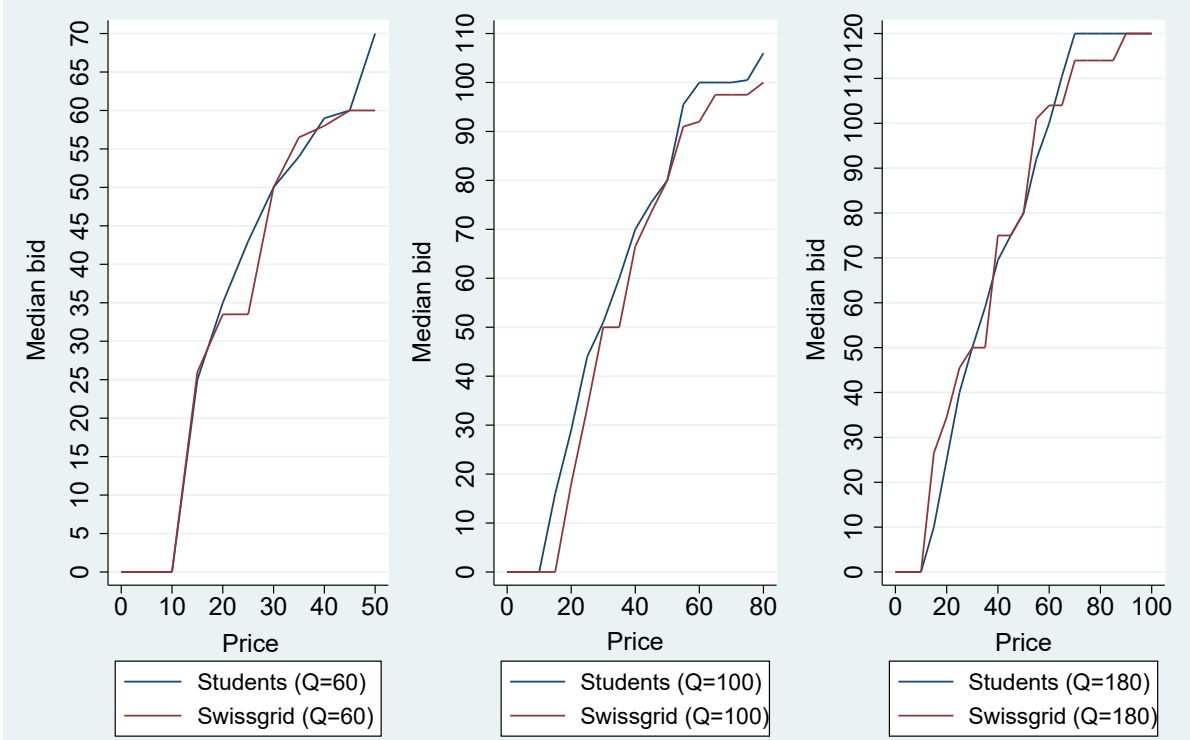

Figure 7: Comparison between practitioner and student bidding

The figure compares the median bids of students to the median bids of Swissgrid practitioners (full information treatment) in case of all demand levels. The figure suggests no notable difference in the average bidding schedule. 